\title{
ENERGY DECAY RATES FOR SOLUTIONS OF THE WAVE EQUATIONS WITH NONLINEAR DAMPING IN EXTERIOR DOMAIN
}

\author{
M. DAOULATLI
}

\begin{abstract}
In this paper we study the behaviors of the energy of solutions of the wave equations with localized nonlinear damping in exterior domains.
\end{abstract}

\section{Introduction and Statement of the Results}

Let $O$ be a compact domain of $\mathbb{R}^{d}(d \geq 1)$ with $C^{\infty}$ boundary $\Gamma$ and $\Omega=\mathbb{R}^{d} \backslash O$. Consider the following wave equation with localized nonlinear damping

$$
\begin{cases}\partial_{t}^{2} u-\Delta u+a(x)\left|\partial_{t} u\right|^{r-1} \partial_{t} u=0 \quad \text { in } \mathbb{R}_{+} \times \Omega, \\ u=0 & \text { on } \mathbb{R}_{+} \times \Gamma, \\ u(0, x)=u_{0} \quad \text { and } \quad \partial_{t} u(0, x)=u_{1}, & \end{cases}
$$

here $\Delta$ denotes the Laplace operator in the space variables. $a(x)$ is a nonnegative function in $L^{\infty}(\Omega)$. Throughout this paper we assume that $1<r \leq 1+\frac{2}{d}$. Below $r_{0}>0$ is a fixed constant such that $O \subset B_{r_{o}}=\left\{x \in \mathbb{R}^{d} ;|x|<r_{0}\right\}$.

The existence and uniqueness of global solutions to the problem (1.1) is standard (see [13]). If $\left(u_{0}, u_{1}\right)$ is in $H_{0}^{1}(\Omega) \cap H^{2}(\Omega) \times H_{0}^{1}(\Omega)$, then the system (1.1), admits a unique solution $u$ in the class

$$
u \in C^{0}\left(\mathbb{R}_{+}, H_{0}^{1}(\Omega) \cap H^{2}(\Omega)\right) \cap C^{1}\left(\mathbb{R}_{+}, H_{0}^{1}(\Omega)\right) .
$$

Let us consider the energy at instant $t$ defined by

$$
E_{u}(t)=\frac{1}{2} \int_{\Omega}\left(|\nabla u(t, x)|^{2}+\left|\partial_{t} u(t, x)\right|^{2}\right) d x .
$$

The energy functional satisfies the following identity

$$
E_{u}(T)+\int_{0}^{T} \int_{\Omega} a(x)\left|\partial_{t} u\right|^{r+1} d x d t=E_{u}(0),
$$

for every $T \geq 0$. Moreover we have

$$
\begin{aligned}
& \left\|\nabla \partial_{t} u\right\|_{L^{\infty}\left(\mathbb{R}_{+}, L^{2}(\Omega)\right)}^{2}+\left\|\partial_{t}^{2} u\right\|_{L^{\infty}\left(\mathbb{R}_{+}, L^{2}(\Omega)\right)}^{2} \\
& \leq 2\left(1+\|a\|_{L^{\infty}}\right)\left(\left\|u_{0}\right\|_{H^{2}}^{2}+\left\|u_{1}\right\|_{H^{1}}^{2}+\left\|u_{1}\right\|_{H^{1}}^{2 r}\right) .
\end{aligned}
$$

Now we give a summary of results on the asymptotic behavior of the energy of solutions of the nonlinear system (1.1) in the free space $\mathbb{R}^{d}$ and for a globally distributed damping. For the Klein Gordon equation a polynomial decay rate is derived by Nakao [16] for compactly

Date: June 6, 2018.

2000 Mathematics Subject Classification. Primary: 35L05, 35B40; Secondary: 35L70, 35B35 .

Key words and phrases. Wave equation, nonlinear damping, Decay rate, exterior domain. 
supported initial data and by Mochizuki and Motai [15] for weighted initial data. More precisely they show that if $1<r<1+\frac{2}{d}$ the energy decays according to

$$
E_{u}(t) \leq C(1+t)^{-\gamma},
$$

where $\gamma<\min \left(1, \frac{2+d-d r}{r-1}\right)$. If $r>1+\frac{2}{d}$, Mochizuki and Motai [15] establishes a complementary non-decay result for a dense set of initial data in $H^{1}\left(\mathbb{R}^{d}\right) \times L^{2}\left(\mathbb{R}^{d}\right)$.

For the wave equation we first quote the result of Ono [19], in which the author consider the wave equation with a damping term equal to $\partial_{t} u+g\left(\partial_{t} u\right)$ where $g$ superlinear and has a polynomial growth. He showed the polynomial decay of the energy. We note that in this case the $L^{2}$ norm of the time derivative on $\mathbb{R}_{+} \times \mathbb{R}^{d}$ of the solution is bounded by the energy of the initial data. Mochizuki and Motai in [15] obtained a logarithmic decay rate when $1<r \leq 1+\frac{2}{d}$ and for a kind of weighted initial data. The corresponding non-decay result in [15] requires $r>1+\frac{2}{d-1}$. Todorova and Yordanov in [23] showed that for compactly supported initial data there exists a positive constant $\tau$ such that $E_{u}(t) \leq C(1+t)^{-\tau}$, when $1<r \leq 1+\frac{2}{d+1}$ and $d \geq 3$. The main idea in this paper is to use the "parabolic" effects coming from the presence of the damping term. Recently, Wakasa and Yordanov in [24] studied the energy decay for dissipative nonlinear wave equations in one space dimension. They established polynomial decay estimates for the energy for compactly supported initial data. More explicetly they show that $E_{u}(t) \leq C(1+t)^{-\tau}$, when $1<r<3$ with $\tau<\min \left(\frac{1}{2}, \frac{3-r}{r-1}\right)$.

In the case of exterior domain we mention the result of Nakao and Jung [18] which consider a dissipation which is allowed to be nonlinear only in a ball, but outside that ball the dissipation must be linear. For the generalized Klein Gordon equation we quote the result of Nakao [17].

For another type of total energy decay property we refer the reader to [10, 11, 21, 1, 20, 9$]$ and references therein.

Before introducing our results we shall state several assumptions:

Hyp A: There exists $L>r_{0}$ such that

$$
a(x) \geq \epsilon_{0}>0 \text { for }|x| \geq L .
$$

Definition 1. Let $\omega$ be an open set of $\Omega$.

(1) $(\omega, T)$ geometrically controls $\Omega$, i.e. every generalized geodesic travelling with speed 1 and issued at $t=0$, enters the set $\omega$ in a time $t<T$.

(2) We say that $\omega$ satisfies $G C C$ if there exists $T>0$ such that $(\omega, T)$ geometrically controls $\Omega$.

This condition is called Geometric Control Condition (see e.g.[2] ). We shall relate the open subset $\omega$ with the damper $a$ by

$$
\omega \subset\left\{x \in \Omega ; a(x)>\epsilon_{0}>0\right\} .
$$

We note that according to [2] and [3] the Geometric Control Condition of Bardos et al is a necessary and sufficient condition for the exponential decay of solutions of the wave equation in bounded domain.

In this paper, we deal with real solutions, the general case can be treated in the same way. Throughout this paper we use the following notations

$$
q(x)=\left(1+|x|^{2}\right)^{\frac{1}{2}}, \text { for } x \in \Omega .
$$


and

$$
p= \begin{cases}2(r+1) & \text { if } d \leq 3 \\ \frac{2 d}{d-2} & \text { if } d \geq 4 .\end{cases}
$$

Now we state the results of this paper.

Theorem 1. We assume that Hyp A holds and $\omega$ satisfies GCC. Let

$$
\begin{array}{ll}
\gamma>0 & \text { if } 1<r<1+\frac{2}{d} \\
0<\gamma<\frac{2}{r-1} & \text { if } r=1+\frac{2}{d} .
\end{array}
$$

Then there exists $C_{0}>0$ such that the following estimate

$$
E_{u}(t) \leq C_{0}(\ln (2+t))^{-\gamma} I_{0}, \text { for all } t \geq 0,
$$

holds for every solution $u$ of (1.1) with initial data $\left(u_{0}, u_{1}\right)$ in $H_{0}^{1}(\Omega) \cap H^{2}(\Omega) \times H_{0}^{1}(\Omega)$, such that

where

$$
\left\|(\ln (1+q))^{\frac{\gamma}{2}} \nabla u_{0}\right\|_{L^{2}}^{2}+\left\|(\ln (1+q))^{\frac{\gamma}{2}} u_{1}\right\|_{L^{2}}^{2}<+\infty,
$$

$$
\begin{aligned}
& I_{0}=\left\|u_{0}\right\|_{H^{2}}^{2}+\left\|u_{1}\right\|_{H^{1}}^{2}+\left\|u_{1}\right\|_{H^{1}}^{2 r}+\left\|u_{0}\right\|_{L^{r+1}}^{r+1}+\left\|(\ln (1+q))^{\frac{\gamma}{2}} \nabla u_{0}\right\|_{L^{2}}^{2} \\
& +\left\|(\ln (1+q))^{\frac{\gamma}{2}} u_{1}\right\|_{L^{2}}^{2}+\left(\left\|u_{0}\right\|_{H^{2}}^{2}+\left\|u_{1}\right\|_{H^{1}}^{2}+\left\|u_{1}\right\|_{H^{1}}^{2 r}\right)^{\frac{p}{2}}+1 .
\end{aligned}
$$

In the result above we see that when $1<r<1+\frac{2}{d}$, we can take any $\gamma>0$, so we expect that we can obtain a rate of decay of the energy for a weight with a polynomial growth.

Theorem 2. We assume that Hyp A holds and $\omega$ satisfies GCC. We suppose that $1<r<$ $1+\frac{2}{d}$. We set

$$
\tau(r, \lambda)=\frac{r \delta_{0}^{r-1}(\lambda+1)^{r-1}(r+1)^{r}}{1+\delta_{0}^{r-1}(\lambda+1)^{r-1}(r+1)^{r}\left(r \delta_{0}^{\frac{r-1}{r}}(\lambda+1)(r+1)+1\right)},
$$

$\lambda$ any positive constant and

$$
\delta_{0}=(\lambda+1)^{\frac{r^{2}}{r^{2}-1}}(r+1)^{-\frac{r}{r-1}} .
$$

We take

$$
\gamma<\min \left(\tau(r, \lambda), \frac{d+2-d r}{r-1}, \frac{p-2 r}{r-1}\right)
$$

and

$$
\alpha(r, \lambda)=\frac{r \delta_{0}^{\frac{r^{2}-1}{r}}(1+\lambda)^{r}(r+1)^{r+1}+1}{\delta_{0}^{r}(r-\tau)(1+\lambda)^{r}(r+1)^{r+1}} .
$$

Then there exists $C_{1}>0$ such that the following estimate

$$
E_{u}(t) \leq C_{1}(1+\alpha t)^{-\gamma} I_{1} \text {, for all } t \geq 0,
$$

holds for every solution $u$ of (1.1) with initial data $\left(u_{0}, u_{1}\right)$ in $H_{0}^{1}(\Omega) \cap H^{2}(\Omega) \times H_{0}^{1}(\Omega)$, such that

where

$$
\left\|(1+\alpha q)^{\frac{\gamma}{2}} \nabla u_{0}\right\|_{L^{2}}^{2}+\left\|(1+\alpha q)^{\frac{\gamma}{2}} u_{1}\right\|_{L^{2}}^{2}<+\infty
$$

$$
\begin{aligned}
& I_{1}=\left\|u_{0}\right\|_{H^{2}}^{2}+\left\|u_{1}\right\|_{H^{1}}^{2}+\left\|u_{1}\right\|_{H^{1}}^{2 r}+\left\|u_{0}\right\|_{L^{r+1}}^{r+1}+\left\|(1+\alpha q)^{\frac{\gamma}{2}} \nabla u_{0}\right\|_{L^{2}}^{2} \\
& +\left\|(1+\alpha q)^{\frac{\gamma}{2}} u_{1}\right\|_{L^{2}}^{2}+\left(\left\|u_{0}\right\|_{H^{2}}^{2}+\left\|u_{1}\right\|_{H^{1}}^{2}+\left\|u_{1}\right\|_{H^{1}}^{2 r}\right)^{\frac{p}{2}}+1 .
\end{aligned}
$$


Remark 1. (1) We note that for a fixed $r$, the best value of $\tau(r, \lambda)$ is obtained when $\lambda$ goes to zero. In addition the function $r \longmapsto \tau(r, 0)=\frac{r}{r+2}$ is increasing on $(1,3]$,

$$
\text { and } \lim _{r \rightarrow 1} \tau(r, 0)=\frac{1}{3} \text {. }
$$

This fact is natural, since the value of $\tau$ is essentially linked to the fact that

$$
\int_{0}^{\infty} \int_{\Omega} a(x)(1+\alpha(q(x)+t))^{\gamma-r-1}|u(t, x)|^{r+1} d x
$$

is finite and we cannot expect to obtain a better value of $\tau$ when $r$ decrease.

(2) The best rate of decay is obtained, when we take

$$
\gamma<\min \left(\frac{r}{r+2}, \frac{d+2-d r}{r-1}, \frac{p-2 r}{r-1}\right) .
$$

(3) We remark that the function $r \longmapsto \alpha(r, 0)$ is decreasing on $(1,3], \lim _{r \rightarrow 1} \alpha(r, 0)=\infty$ and $\alpha(3,0) \geq \frac{10}{3}$.

The case of initial data with compact support

Theorem 3. We assume that Hyp A holds and $\omega$ satisfies GCC. We suppose that $1<r<$ $1+\frac{2}{d}$. We set

$$
\tau_{1}(r, \lambda)=\frac{2 r \delta_{0}^{r-1}(\lambda+1)^{r-1}(r+1)^{r}}{1+\delta_{0}^{r-1}(\lambda+1)^{r-1}(r+1)^{r}\left(r \delta_{0}^{\frac{r-1}{r}}(\lambda+1)(r+1)+2\right)},
$$

$\lambda$ any positive constant and

$$
\delta_{0}=(\lambda+1)^{\frac{r^{2}}{r^{2}-1}}(r+1)^{-\frac{r}{r-1}}
$$

We take

$$
\gamma<\min \left(\tau_{1}(r, \lambda), \frac{d+2-d r}{r-1}, \frac{p-2 r}{r-1}\right)
$$

and

$$
\alpha(r, \lambda)=\frac{r \delta_{0}^{\frac{r^{2}-1}{r}}(1+\lambda)^{r}(r+1)^{r+1}+1}{\delta_{0}^{r}\left(r-\tau_{1}\right)(1+\lambda)^{r}(r+1)^{r+1}} .
$$

Then there exists $C_{1}>0$ such that the following estimate

$$
E_{u}(t) \leq C_{1}\left(\frac{R}{R+\alpha t}\right)^{\gamma} I_{2}, \text { for all } t \geq 0,
$$

holds for every solution $u$ of (1.1) with initial data $\left(u_{0}, u_{1}\right)$ in $H_{0}^{1}(\Omega) \cap H^{2}(\Omega) \times H_{0}^{1}(\Omega)$ such that the support of the initial data is contained in $B_{R}$, where

$$
\begin{aligned}
& I_{2}=\left\|u_{0}\right\|_{H^{2}}^{2}+\left\|u_{1}\right\|_{H^{1}}^{2}+\left\|u_{1}\right\|_{H^{1}}^{2 r}+\left\|u_{0}\right\|_{L^{r+1}}^{r+1} \\
& +\left(\left\|u_{0}\right\|_{H^{2}}^{2}+\left\|u_{1}\right\|_{H^{1}}^{2}+\left\|u_{1}\right\|_{H^{1}}^{2 r}\right)^{\frac{p}{2}}+1 .
\end{aligned}
$$

Remark 2. (1) Our results are also valid for the case $\Omega=\mathbb{R}^{d}, d \geq 3$, where the boundary condition is dropped.

(2) We note that for a fixed $r$, the best value of $\tau_{1}(r, \lambda)$ is obtained when $\lambda$ goes to zero. In addition the function $r \longmapsto \tau_{1}(r, 0)=\frac{2 r}{r+3}$ is increasing on $(1,3]$,

$$
\text { and } \lim _{r \rightarrow 1} \tau_{1}(r, 0)=\frac{1}{2} \text {. }
$$


This fact is natural, since the value of $\tau_{1}$ is essentially linked to the fact that

$$
\int_{0}^{\infty} \int_{\Omega} a(x)(R+\alpha t)^{\gamma-r-1}|u(t, x)|^{r+1} d x
$$

is finite, which depends on the behavior of

$$
\int_{\Omega} a(x)|u(t, x)|^{r+1} d x
$$

therefore we cannot expect to obtain a better value of $\tau_{1}$ when $r$ decrease.

(3) The best rate of decay is obtained, when we take

$$
\gamma<\min \left(\frac{2 r}{r+3}, \frac{d+2-d r}{r-1}, \frac{p-2 r}{r-1}\right) .
$$

When $d=1$, we obtain that

$$
\begin{array}{cl}
1 / 2<\gamma<\frac{2 r}{r+3} & \text { if } 1<r \leq \frac{2}{3} \sqrt{7}+\frac{1}{3} \\
\gamma<\frac{3-r}{r-1} & \text { if } \frac{2}{3} \sqrt{7}+\frac{1}{3}<r<3 .
\end{array}
$$

Our decay rates is better or equal than the one obtained by Wakasa and Yordanov in [24].

(4) We remark that the function $r \longmapsto \alpha(r, 0)$ is decreasing on $(1,3], \lim _{r \rightarrow 1} \alpha(r, 0)=\infty$ and $\alpha(3,0) \geq 18$. In addition we have

$$
\alpha(r, \lambda) \geq \alpha(r, 0), \text { for all }(r, \lambda) \in(1,3] \times \mathbb{R}_{+}^{*},
$$

so the case $r=1$ cannot be obtained by letting $r$ goes to 1 .

The main difficulty in establishing such results is the lack of control of the $L^{2}$ norm of the solution. This is an essential difference with the equation in a bounded domain or the Klein-Gordon equation or in the case of unbounded domain with finite measure [4]. The other difficulties is that the $L^{2}$ norm of the time derivative on $\mathbb{R}_{+} \times \Omega$ is not controlled by the initial energy and the fact that the domain is with infinite measure.

To prove our results it is sufficient to show the integrability of $\varphi^{\prime} E_{u}$ over $(0, \infty)$. For this purpose we show an estimate on a functional $X(t)$ which control the weighted energy functional (see for example [7] and [8] for similar idea). Also we prove a weighted observability estimate for the local energy of solutions the wave equation with external force.

The rest of the paper is organized as follows. In section 2 we present some results on the weighted energy and we give a weighted observability estimate for the local energy. The section 3 is devoted to the proof of theorem 1 and in section 4 we give the proof of theorem 2. In the last section we give the needed results to show the theorem 3 .

\section{Weighted observabiLity estimate}

The next result concern the weighted energy estimate for solutions of (1.1) with initial data with finite weighted energy.

Proposition 1. Let $\varphi$ be a positive function in $C^{2}\left(\mathbb{R}_{+}\right)$such that $\varphi^{\prime}$ and $\varphi^{\prime \prime}$ are in $L^{\infty}\left(\mathbb{R}_{+}\right)$. Let $u$ be a solution of (1.1) with initial data $\left(u_{0}, u_{1}\right)$ in $H_{0}^{1}(\Omega) \cap H^{2}(\Omega) \times H_{0}^{1}(\Omega)$. We set

$$
E_{\varphi}(u)(t)=\frac{1}{2} \int_{\Omega} \varphi(\eta q(x)+\alpha t)\left(|\nabla u|^{2}+\left|\partial_{t} u\right|^{2}\right) d x .
$$




$$
\begin{aligned}
& \text { If } E_{\varphi}(u)(0)<\infty \text {, then } \\
& \qquad \sqrt{\varphi} \nabla u \in L_{\text {loc }}^{\infty}\left(\mathbb{R}_{+},\left(L^{2}(\Omega)\right)^{d}\right) \text { and } \sqrt{\varphi} \partial_{t} u \in L_{l o c}^{\infty}\left(\mathbb{R}_{+}, L^{2}(\Omega)\right) .
\end{aligned}
$$

Moreover, we have

$$
\begin{aligned}
& E_{\varphi}(u)(t+T)+\int_{t}^{t+T} \int_{\Omega} a(x) \varphi(s, x)\left|\partial_{t} u\right|^{r+1} d x d s \\
& \leq E_{\varphi}(u)(t)+\frac{\alpha+\eta}{2} \int_{t}^{t+T} \int_{\Omega}\left|\varphi^{\prime}(s, x)\right|\left(|\nabla u(s)|^{2}+\left|\partial_{t} u(s)\right|^{2}\right) d x d s .
\end{aligned}
$$

for every $t \geq 0$ and $T \geq 0$, where $\varphi^{(j)}(t, x)=\varphi^{(j)}(\eta q(x)+\alpha t)$, for $j=0,1,2$ and $\alpha, \eta \geq 0$. Proof. The first step is to show (2.2) . Let $n \in \mathbb{N}^{*}$. We define

$$
g_{n}(s)=g \circ\left(I+n^{-1} g\right)^{-1}(s)=n\left(s-\left(I+n^{-1} g\right)^{-1}(s)\right),
$$

the Yosida approximation of $g: s \longmapsto|s|^{r-1} s . g_{n}$ is monotone increasing, globally Lipschitz and $g_{n}(0)=0$. Let $w$ be the solution of the following system

$$
\begin{cases}\partial_{t}^{2} w-\Delta w+a(x)(1+\varphi)^{\frac{1}{2}} g_{n}\left((1+\varphi)^{-\frac{1}{2}} \partial_{t} w+h(t, x)\right)=f(t, x) & \mathbb{R}_{+} \times \Omega \\ w=0 & \mathbb{R}_{+} \times \partial \Omega \\ \left(w(0), \partial_{t} w(0)\right)=\left(w_{0}, w_{1}\right) \in H_{D}(\Omega) \times L^{2}(\Omega) & \end{cases}
$$

with $f \in L_{\text {loc }}^{2}\left(\mathbb{R}_{+}, L^{2}(\Omega)\right)$ and $(1+\varphi)^{\frac{1}{2}} h \in L_{\text {loc }}^{2}\left(\mathbb{R}_{+}, L^{2}(\Omega)\right)$, where $H_{D}(\Omega)$ the completion of $C_{c}^{\infty}(\Omega)$ with respect to the norm

$$
\left\|\varphi_{0}\right\|_{H_{D}}^{2}=\int_{\Omega}\left|\nabla \varphi_{0}\right|^{2} d x
$$

$g_{n}$ is a global Lipschitz. function, therefore

$$
a(x)(1+\varphi)^{\frac{1}{2}} g_{n}\left((1+\varphi)^{-\frac{1}{2}} \partial_{t} w+h\right) \in L_{l o c}^{2}\left(\mathbb{R}_{+}, L^{2}(\Omega)\right) .
$$

Using the fact that the function $f \in L_{l o c}^{2}\left(\mathbb{R}_{+}, L^{2}(\Omega)\right)$, we infer that the unique solution of (2.4)

$$
w \in C\left(\mathbb{R}_{+}, H_{D}(\Omega)\right) \text { and } \partial_{t} w \in C\left(\mathbb{R}_{+}, L^{2}(\Omega)\right),
$$

and the following energy identity

$$
\begin{aligned}
& E_{w}(t)+\int_{0}^{t} \int_{\Omega} a(x)(1+\varphi)^{\frac{1}{2}} g_{n}\left((1+\varphi)^{-\frac{1}{2}} \partial_{t} w+h(s, x)\right) \partial_{t} w d x d s \\
= & E_{w}(0)+\int_{0}^{t} \int_{\Omega} f(s, x) \partial_{t} w d x d s
\end{aligned}
$$

holds for every $t \geq 0$.

Let $u_{n}$ be the solution of the following system

$$
\begin{cases}\partial_{t}^{2} u_{n}-\Delta u_{n}+a(x) g_{n}\left(\partial_{t} u_{n}\right)=0 & \mathbb{R}_{+} \times \Omega \\ u_{n}=0 & \mathbb{R}_{+} \times \partial \Omega \\ \left(u_{n}(0), \partial_{t} u_{n}(0)\right)=\left(u_{0}, u_{1}\right) & \end{cases}
$$

with $\left(u_{0}, u_{1}\right)$ in $H_{0}^{1}(\Omega) \cap H^{2}(\Omega) \times H_{0}^{1}(\Omega)$ such that

$$
\int_{\Omega} \varphi(\eta q(x))\left(\left|\nabla u_{0}\right|^{2}+\left|u_{1}\right|^{2}\right) d x<\infty .
$$


The function $g_{n}$ is globally Lipschitz., hence

$$
u_{n} \in L^{\infty}\left([0, T], H_{0}^{1}(\Omega) \cap H^{2}(\Omega)\right) \cap W^{1, \infty}\left([0, T], H_{0}^{1}(\Omega)\right),
$$

moreover we have the following energy identity

$$
E_{u_{n}}(t)+\int_{0}^{t} \int_{\Omega} a(x) g_{n}\left(\partial_{t} u_{n}\right) \partial_{t} u_{n} d x d s=E_{u_{n}}(0) .
$$

In addition we have

$$
\begin{aligned}
& \left\|\Delta u_{n}\right\|_{L^{\infty}\left(\mathbb{R}_{+}, L^{2}(\Omega)\right)}^{2}+\left\|\nabla \partial_{t} u_{n}\right\|_{L^{\infty}\left(\mathbb{R}_{+}, L^{2}(\Omega)\right)}^{2}+\left\|\partial_{t}^{2} u_{n}\right\|_{L^{\infty}\left(\mathbb{R}_{+}, L^{2}(\Omega)\right)}^{2} \\
& \leq 2\left(1+\|a\|_{L^{\infty}}\right)\left(\left\|u_{0}\right\|_{H^{2}}^{2}+\left\|u_{1}\right\|_{H^{1}}^{2}\right) .
\end{aligned}
$$

From (2.8) and (2.9), we infer that there exists $u$ and $\psi$ in $L^{\frac{r+1}{r}}((0, T) \times \Omega, a)$ such that

$u_{n} \underset{n \rightarrow+\infty}{\longrightarrow} u$ in the weak star topology of $L^{\infty}\left([0, T], H_{0}^{1}(\Omega) \cap H^{2}(\Omega)\right)$

$\partial_{t} u_{n} \underset{n \rightarrow+\infty}{\longrightarrow} \partial_{t} u$ in the weak star topology of $L^{\infty}\left([0, T], H_{0}^{1}(\Omega)\right)$

$\left(I+n^{-1} g\right)^{-1}\left(\partial_{t} u_{n}\right) \underset{n \rightarrow+\infty}{\longrightarrow} \partial_{t} u$ in the weak topology of $L^{r+1}((0, T) \times \Omega, a)$

$g_{n}\left(\partial_{t} u_{n}\right) \underset{n \rightarrow+\infty}{\longrightarrow} \psi$ in the weak topology of $L^{\frac{r+1}{r}}((0, T) \times \Omega, a)$,

where

$$
L^{r+1}((0, T) \times \Omega, a)=\left\{\varkappa \text { such that } \int_{0}^{T} \int_{\Omega}|\varkappa(s, x)|^{r+1} a(x) d x d s<\infty\right\} .
$$

To show that, $\psi=g\left(\partial_{t} u\right)$, we proceed as in [13, P55-56]. By a classical compactness argument, we can show that there exists a subsequence of $\left(u_{n}\right)$ still denoted by $\left(u_{n}\right)$, such that

$$
\partial_{t} u_{n} \underset{n \rightarrow+\infty}{\longrightarrow} \partial_{t} u \text { strongly in } L^{2}(K)
$$

for a given compact subset $K$ of $(0, T) \times \Omega$. Therefore we can assume that

$$
\partial_{t} u_{n} \underset{n \rightarrow+\infty}{\longrightarrow} \partial_{t} u \text {, a.e. in } K \text {. }
$$

Since the function $s \longmapsto\left(I+n^{-1} g\right)^{-1}(s)$, is non-expansive on $\mathbb{R}$, we obtain

$$
\left(I+n^{-1} g\right)^{-1}\left(\partial_{t} u_{n}\right) \underset{n \rightarrow+\infty}{\longrightarrow} \partial_{t} u \text {, a.e. in } K \text {. }
$$

Hence

$$
g_{n}\left(\partial_{t} u_{n}\right) \underset{n \rightarrow+\infty}{\longrightarrow} g\left(\partial_{t} u\right) \text {, a.e. in } K \text {. }
$$

This enough to gives $\psi=g\left(\partial_{t} u\right)$. Therefore $u$ is a solution of (1.1) with initial data in $H_{0}^{1}(\Omega) \cap H^{2}(\Omega) \times H_{0}^{1}(\Omega)$ such that

$$
\int_{\Omega} \varphi(\eta q(x))\left(\left|\nabla u_{0}\right|^{2}+\left|u_{1}\right|^{2}\right) d x<\infty .
$$

We set $v_{n}=(1+\varphi)^{\frac{1}{2}} u_{n}$. Therefore $v_{n}$ satisfies

$$
\begin{cases}\partial_{t}^{2} v_{n}-\Delta v_{n}+a(x)(1+\varphi)^{\frac{1}{2}} g_{n}\left((1+\varphi)^{-\frac{1}{2}} \partial_{t} v_{n}+h_{n}(t, x)\right)=f(t, x) & \mathbb{R}_{+} \times \Omega \\ v_{n}=0 & \mathbb{R}_{+} \times \partial \Omega \\ \left(v_{n}(0), \partial_{t} v_{n}(0)\right)=\left(v_{0}, v_{1}\right) & \end{cases}
$$


with

$$
\begin{aligned}
& \left(v_{0}, v_{1}\right)=\left((1+\varphi(\eta q(x)))^{\frac{1}{2}} u_{0}, \frac{1}{2}(1+\varphi(\eta q(x)))^{-\frac{1}{2}} \varphi^{\prime}(\eta q(x)) \eta u_{0}+(1+\varphi(\eta q(x)))^{\frac{1}{2}} u_{1}\right) \\
& h_{n}=-\frac{\alpha}{2}(1+\varphi)^{-1} \varphi^{\prime} u_{n}
\end{aligned}
$$

and

$$
\begin{aligned}
& f=\frac{1}{2}(1+\varphi)^{-\frac{1}{2}}\left[\eta^{2}\left(\varphi^{\prime \prime}-\frac{1}{2}\left(\varphi^{\prime}\right)^{2}(1+\varphi)^{-1}\right) \frac{|x|^{2}}{q^{2}}+\eta\left(\frac{d}{q}-\frac{|x|^{2}}{q^{3}}\right) \varphi^{\prime}\right] u_{n} \\
& +\frac{\alpha^{2}}{2}(1+\varphi)^{-\frac{1}{2}}\left[\varphi^{\prime \prime}-\frac{1}{2}\left(\varphi^{\prime}\right)^{2}(1+\varphi)^{-1}\right] u_{n}+\varphi^{\prime}(1+\varphi)^{-\frac{1}{2}}\left(\alpha \partial_{t} u_{n}+\eta \frac{x \cdot \nabla u_{n}}{q}\right) .
\end{aligned}
$$

Hence, recalling (2.7), $\varphi^{\prime} \in L^{\infty}\left(\mathbb{R}_{+}\right)$and $\varphi^{\prime \prime} \in L^{\infty}\left(\mathbb{R}_{+}\right)$

$$
\begin{aligned}
& \left(v_{0}, v_{1}\right) \in H_{D}(\Omega) \times L^{2}(\Omega) \\
& (1+\varphi)^{\frac{1}{2}} h_{n} \in L_{l o c}^{2}\left(\mathbb{R}_{+}, L^{2}(\Omega)\right) \\
& f \in L_{l o c}^{2}\left(\mathbb{R}_{+}, L^{2}(\Omega)\right) .
\end{aligned}
$$

Therefore using (2.5) along with

$$
(1+\varphi)^{-\frac{1}{2}} \partial_{t} v_{n}-\frac{\alpha}{2}(1+\varphi)^{-1} \varphi^{\prime} u_{n}=\partial_{t} u_{n}
$$

and making some arrangement, we deduce that

$$
\begin{aligned}
& E_{v_{n}}(t)+\int_{0}^{t} \int_{\Omega} a(x)(1+\varphi) g_{n}\left(\partial_{t} u_{n}\right) \partial_{t} u_{n} d x d s \\
& =E_{v_{n}}(0)+\int_{0}^{t} \int_{\Omega}(1+\varphi)^{\frac{1}{2}} f(s, x) \partial_{t} u_{n} d x d s+\frac{\alpha}{2} \int_{0}^{t} \int_{\Omega}(1+\varphi)^{-\frac{1}{2}} f(s, x) \varphi^{\prime} u_{n} d x d s \\
& -\frac{\alpha}{2} \int_{0}^{t} \int_{\Omega} a(x) \varphi^{\prime} g_{n}\left(\partial_{t} u_{n}\right) u_{n} d x d s .
\end{aligned}
$$

On the other hand, since $\varphi^{\prime} \in L^{\infty}\left(\mathbb{R}_{+}\right)$and $\varphi^{\prime \prime} \in L^{\infty}\left(\mathbb{R}_{+}\right)$then there exists a positive constant $C=C(\varphi)$ such that

$$
\begin{aligned}
& \left|\int_{0}^{t} \int_{\Omega}(1+\varphi)^{\frac{1}{2}} f(s, x) \partial_{t} u_{n} d x d s\right| \leq C \int_{0}^{t} \int_{\Omega}\left|u_{n}\right|^{2}+\left|\partial_{t} u_{n}\right|^{2}+\left|\nabla u_{n}\right|^{2} d x d s, \\
& \left|\frac{\alpha}{2} \int_{0}^{t} \int_{\Omega}(1+\varphi)^{-\frac{1}{2}} f(s, x) \varphi^{\prime} u_{n} d x d s\right| \leq C \int_{0}^{t} \int_{\Omega}\left|u_{n}\right|^{2}+\left|\partial_{t} u_{n}\right|^{2}+\left|\nabla u_{n}\right|^{2} d x d s .
\end{aligned}
$$

To estimate the last term of the RHS of (2.14), we use Young's inequality along with the fact that $g(s)=|s|^{r-1} s$

$\left|\frac{\alpha}{2} \int_{0}^{t} \int_{\Omega} a(x) \varphi^{\prime} g_{n}\left(\partial_{t} u_{n}\right) u_{n} d x d s\right| \leq C \int_{0}^{t} \int_{\Omega} a(x)\left|u_{n}\right|^{r+1}+a(x)\left|\left(I+n^{-1} g\right)^{-1}\left(\partial_{t} u_{n}\right)\right|^{r+1} d x d s$.

Now using (2.8) and the fact that

$$
g_{n}\left(\partial_{t} u_{n}\right) \partial_{t} u_{n} \geq\left|\left(I+n^{-1} g\right)^{-1}\left(\partial_{t} u_{n}\right)\right|^{r+1},
$$

we infer that

and

$$
\int_{0}^{t} \int_{\Omega} a(x)\left|\left(I+n^{-1} g\right)^{-1}\left(\partial_{t} u_{n}\right)\right|^{r+1} d x d s \leq E_{u_{n}}(0),
$$

$$
\int_{0}^{t} \int_{\Omega}\left|\partial_{t} u_{n}\right|^{2}+\left|\nabla u_{n}\right|^{2} d x d s \leq(1+t) E_{u_{n}}(0) .
$$


We have

Therefore

$$
\begin{aligned}
& \int_{\Omega}\left|u_{n}(s)\right|^{2} d x \leq C(1+s)\left(E_{u_{n}}(0)+\left\|u_{0}\right\|_{L^{2}}^{2}\right) \\
& \text { and } \\
& \int_{\Omega}\left|u_{n}(s)\right|^{r+1} d x \leq C(1+s)^{\frac{r+1}{2}}\left(E_{u_{n}}(0)+\left\|u_{0}\right\|_{L^{2}}^{2}\right)^{\frac{r+1}{2}} .
\end{aligned}
$$

$$
\begin{aligned}
& \int_{0}^{t} \int_{\Omega}\left|u_{n}\right|^{2} d x d s \leq C(1+t)^{2}\left(E_{u_{n}}(0)+\left\|u_{0}\right\|_{L^{2}}^{2}\right), \\
& \int_{0}^{t} \int_{\Omega}\left|u_{n}\right|^{r+1} d x d s \leq C(1+t)^{\frac{r+3}{2}}\left(E_{u_{n}}(0)+\left\|u_{0}\right\|_{L^{2}}^{2}\right)^{\frac{r+1}{2}} .
\end{aligned}
$$

Combining the estimates above with (2.14), we obtain

$$
\begin{aligned}
& E_{v_{n}}(t)+\int_{0}^{t} \int_{\Omega} a(x)(1+\varphi) g_{n}\left(\partial_{t} u_{n}\right) \partial_{t} u_{n} d x d s \\
& \leq C(1+t)^{3}\left(E_{v_{n}}(0)+E_{u_{n}}(0)+\left(E_{u_{n}}(0)+\left\|u_{0}\right\|_{L^{2}}^{2}\right)^{\frac{r+1}{2}}+\left\|u_{0}\right\|_{L^{2}}^{2}\right) .
\end{aligned}
$$

It is easy to see that

$$
E_{\varphi}\left(u_{n}\right)(t) \leq 2 E_{v_{n}}(t)+C\left\|u_{n}(t)\right\|_{L^{2}}^{2} .
$$

Therefore combining the estimate above with (2.17) and (2.16) we obtain

$$
\begin{aligned}
& E_{\varphi}\left(u_{n}\right)(t)+\int_{0}^{t} \int_{\Omega} a(x)(1+\varphi) g_{n}\left(\partial_{t} u_{n}\right) \partial_{t} u_{n} d x d s \\
& \leq C(1+t)^{2}\left(E_{\varphi}\left(u_{n}\right)(0)+\left\|u_{0}\right\|_{L^{2}}^{2}+E_{u_{n}}(0)+\left(E_{u_{n}}(0)+\left\|u_{0}\right\|_{L^{2}}^{2}\right)^{\frac{r+1}{2}}\right) .
\end{aligned}
$$

Note that in the estimate above we have used the fact that

$$
E_{v_{n}}(0) \leq E_{\varphi}\left(u_{n}\right)(0)+\left\|u_{0}\right\|_{L^{2}}^{2} .
$$

Now using (2.18) and (2.15), we infer that

$$
\begin{aligned}
& \sqrt{1+\varphi} \partial_{x_{i}} u_{n} \underset{n \rightarrow+\infty}{\longrightarrow} \psi_{i} \text { in the weak star topology of } L^{\infty}\left([0, T], L^{2}(\Omega)\right), i \in\{1, . ., d\} \\
& \sqrt{1+\varphi} \partial_{t} u_{n} \underset{n \rightarrow+\infty}{\longrightarrow} \phi_{1} \text { in the weak star topology of } L^{\infty}\left([0, T], L^{2}(\Omega)\right) \\
& (a(1+\varphi))^{\frac{1}{r+1}}\left(I+n^{-1} g\right)^{-1}\left(\partial_{t} u_{n}\right) \underset{n \rightarrow+\infty}{\longrightarrow} \phi_{2} \text { in the weak topology of } L^{r+1}((0, T) \times \Omega) .
\end{aligned}
$$

Now we show that

$$
\psi_{i}=\sqrt{1+\varphi} \partial_{x_{i}} u, \phi_{1}=\sqrt{1+\varphi} \partial_{t} u \text { and } \phi_{2}=(a(1+\varphi))^{\frac{1}{r+1}} \partial_{t} u .
$$

Let $K$ be a compact set of $(0, T) \times \Omega$. Using (2.12), we get

$$
\sqrt{1+\varphi} \partial_{t} u_{n} \underset{n \rightarrow+\infty}{\longrightarrow} \sqrt{1+\varphi} \partial_{t} u \text {, a.e. in } K,
$$

and using the fact that the function $s \longmapsto\left(I+n^{-1} g\right)^{-1}(s)$, is non-expansive on $\mathbb{R}$, we obtain

$$
(a(1+\varphi))^{\frac{1}{r+1}}\left(I+n^{-1} g\right)^{-1}\left(\partial_{t} u_{n}\right) \underset{n \rightarrow+\infty}{\longrightarrow}(a(1+\varphi))^{\frac{1}{r+1}} \partial_{t} u \text {, a.e. in } K,
$$

This is enough to imply

$$
\phi_{1}=\sqrt{1+\varphi} \partial_{t} u \text { and } \phi_{2}=(a(1+\varphi))^{\frac{1}{r+1}} \partial_{t} u .
$$


From (2.10) and by a classical compactness argument, we can show that there exists a subsequence of $\left(u_{n}\right)$ still denoted by $\left(u_{n}\right)$, such that

$$
\partial_{x_{i}} u_{n} \underset{n \rightarrow+\infty}{\longrightarrow} \partial_{x_{i}} u \text { strongly in } L^{2}(K) .
$$

Therefore extracting a subsequence if necessary

$$
\partial_{x_{i}} u_{n} \underset{n \rightarrow+\infty}{\longrightarrow} \partial_{x_{i}} u \text {, a.e. in } K
$$

which gives

$$
\sqrt{1+\varphi} \partial_{x_{i}} u_{n} \underset{n \rightarrow+\infty}{\longrightarrow} \sqrt{1+\varphi} \partial_{x_{i}} u \text {, a.e. in } K \text {. }
$$

We conclude that

$$
\psi_{i}=\sqrt{1+\varphi} \partial_{x_{i}} u, i \in\{1, . ., d\}
$$

Therefore

$$
\sqrt{\varphi} \nabla u \in L_{\text {loc }}^{\infty}\left(\mathbb{R}_{+},\left(L^{2}(\Omega)\right)^{d}\right) \text { and } \sqrt{\varphi} \partial_{t} u \in L_{l o c}^{\infty}\left(\mathbb{R}_{+}, L^{2}(\Omega)\right) .
$$

Now we will prove the energy estimate (2.3) . We remind that

$$
u \in L_{l o c}^{\infty}\left(\mathbb{R}_{+}, H_{0}^{1}(\Omega) \cap H^{2}(\Omega)\right) \cap W^{1, \infty}\left(\mathbb{R}_{+}, H_{0}^{1}(\Omega)\right) \cap W^{2, \infty}\left(\mathbb{R}_{+}, L^{2}(\Omega)\right) .
$$

Let $R \gg 1$ and setting $S(R)=\partial B_{R}$. It is easy to see that

$$
\begin{aligned}
& \frac{1}{2} \frac{d}{d t} \int_{\Omega \cap B_{R}} \varphi\left(|\nabla u(t)|^{2}+\left|\partial_{t} u(t)\right|^{2}\right) d x+\int_{\Omega \cap B_{R}} a(x) \varphi\left|\partial_{t} u(t)\right|^{r+1} d x \\
& =\frac{\alpha}{2} \int_{\Omega \cap B_{R}} \varphi^{\prime}\left(|\nabla u(t)|^{2}+\left|\partial_{t} u(t)\right|^{2}\right) d x+\int_{\Omega \cap B_{R}} \varphi \nabla u(t) \cdot \nabla \partial_{t} u(t)+\varphi \partial_{t} u(t) \partial_{t}^{2} u(t) d x \\
& +\int_{\Omega \cap B_{R}} a(x) \varphi\left|\partial_{t} u(t)\right|^{r+1} d x \\
& =\frac{\alpha}{2} \int_{\Omega \cap B_{R}} \varphi^{\prime}\left(|\nabla u(t)|^{2}+\left|\partial_{t} u(t)\right|^{2}\right) d x+\int_{\Omega \cap B_{R}} \nabla u(t) \cdot \nabla\left(\varphi \partial_{t} u(t)\right)+\varphi \partial_{t} u(t) \partial_{t}^{2} u(t) d x \\
& +\int_{\Omega \cap B_{R}} a(x) \varphi\left|\partial_{t} u(t)\right|^{r+1} d x-\eta \int_{\Omega \cap B_{R}} \varphi^{\prime} \frac{x \cdot \nabla u(t)}{q(x)} \partial_{t} u(t) d x .
\end{aligned}
$$

Green's formula along with the fact that $u$ is a solution of (1.1),

$$
\begin{aligned}
& \frac{1}{2} \frac{d}{d t} \int_{\Omega \cap B_{R}} \varphi\left(|\nabla u(t)|^{2}+\left|\partial_{t} u(t)\right|^{2}\right) d x+\int_{\Omega \cap B_{R}} a(x) \varphi\left|\partial_{t} u(t)\right|^{r+1} d x \\
& =\frac{\alpha}{2} \int_{\Omega \cap B_{R}} \varphi^{\prime}\left(|\nabla u(t)|^{2}+\left|\partial_{t} u(t)\right|^{2}\right) d x-\eta \int_{\Omega \cap B_{R}} \varphi^{\prime} \frac{x \cdot \nabla u(t)}{q(x)} \partial_{t} u(t) d x+\int_{S(R)} \varphi \frac{x \cdot \nabla u(t)}{R} \partial_{t} u(t) d S .
\end{aligned}
$$

Integrating the estimate above between $t$ and $t+T$, we obtain

$$
\begin{aligned}
& \int_{\Omega \cap B_{R}} \varphi\left(|\nabla u(t+T)|^{2}+\left|\partial_{t} u(t+T)\right|^{2}\right) d x+\int_{t}^{t+T} \int_{\Omega \cap B_{R}} a(x) \varphi\left|\partial_{t} u\right|^{r+1} d x d s \\
& \leq E_{\varphi}(u)(t)+\frac{\alpha}{2} \int_{t}^{t+T} \int_{\Omega}\left|\varphi^{\prime}\right|\left(|\nabla u(s)|^{2}+\left|\partial_{t} u(s)\right|^{2}\right) d x d s \\
& +\eta \int_{t}^{t+T} \int_{\Omega}\left|\varphi^{\prime} \frac{x \cdot \nabla u(s)}{q(x)} \partial_{t} u(s)\right| d x d s+\int_{t}^{t+T} \int_{S(R)} \varphi\left|\frac{x \cdot \nabla u(s)}{R} \partial_{t} u(s)\right| d S d s .
\end{aligned}
$$

Using Young's inequality

$$
\int_{t}^{t+T} \int_{S(R)} \varphi\left|\frac{x \cdot \nabla u}{R} \partial_{t} u\right| d S d \tau \leq \frac{1}{2} \int_{t}^{t+T} \int_{S(R)}\left(\left|\partial_{r} u\right|^{2}+\left|\partial_{t} u\right|^{2}\right) \varphi d S d \tau .
$$


From (2.19), we infer that

$$
\liminf _{R \longrightarrow+\infty} \int_{t}^{t+T} \int_{S(R)} \varphi\left|\frac{x \cdot \nabla u}{R} \partial_{t} u\right| d S d \tau=0 .
$$

Passing to the limit in (2.20), we get

$$
\begin{aligned}
& E_{\varphi}(u)(t+T)+\int_{t}^{t+T} \int_{\Omega} a(x) \varphi\left|\partial_{t} u\right|^{r+1} d x d s \leq E_{\varphi}(u)(t) \\
& +\frac{\alpha}{2} \int_{t}^{t+T} \int_{\Omega}\left|\varphi^{\prime}\right|\left(|\nabla u(s)|^{2}+\left|\partial_{t} u(s)\right|^{2}\right) d x d s+\eta \int_{t}^{t+T} \int_{\Omega}\left|\varphi^{\prime} \frac{x \cdot \nabla u}{q(x)} \partial_{t} u(s)\right| d x d s
\end{aligned}
$$

Young's inequality, gives

$$
\begin{aligned}
& E_{\varphi}(u)(t+T)+\int_{t}^{t+T} \int_{\Omega} a(x) \varphi\left|\partial_{t} u\right|^{r+1} d x d s \\
& \leq E_{\varphi}(u)(t)+\frac{\alpha+\eta}{2} \int_{t}^{t+T^{2}} \int_{\Omega}\left|\varphi^{\prime}\right|\left(|\nabla u(s)|^{2}+\left|\partial_{t} u(s)\right|^{2}\right) d x d s .
\end{aligned}
$$

The proof of our results need a weighted observability estimate for the local energy and to show such result we need to prove a unique continuation result for the wave equation.

Lemma 1. We assume that Hyp A holds and $(\omega, T)$ geometrically controls $\Omega$. Then the only solution of the system

in the class

$$
\begin{cases}\partial_{t}^{2} z-\Delta z=0 & \text { in }(0, T) \times \Omega \\ z=0 & \text { on }(0, T) \times \Gamma \\ a(x) \partial_{t} z=0 & \text { on }(0, T) \times \Omega\end{cases}
$$

$$
C^{0}\left([0, T] ; H_{D}(\Omega)\right) \cap C^{1}\left([0, T] ; L^{2}(\Omega)\right),
$$

is the null one, where $H_{D}(\Omega)$ is the completion of $C_{c}^{\infty}(\Omega)$ with respect to the norm

$$
\|\varphi\|_{H}^{2}=\int_{\Omega}|\nabla \varphi(x)|^{2} d x .
$$

Proof. Let $\chi \in C_{c}^{\infty}\left(\mathbb{R}^{d}\right)$ such that $\chi=1$ on $\{|x| \leq L\}$ and the support of $\chi$ is contained in $\{|x| \leq 2 L\}$. First we note that $H_{D}(\Omega) \subset H_{l o c}^{1}(\Omega)$. Let $z$ be a solution of the system (2.21) . We set $w=\chi z$, we observe that

$$
\begin{cases}\partial_{t}^{2} w-\Delta w=-2 \nabla \chi \nabla z-z \Delta \chi & \text { in }(0, T) \times \Omega \cap B_{2 L}, \\ w=0 & \text { on }(0, T) \times \Gamma \cup\{|x|=2 L\}, \\ \left(w_{0}, w_{1}\right) \in H_{0}^{1}\left(\Omega \cap B_{2 L}\right) \times L^{2}\left(\Omega \cap B_{2 L}\right) & \\ a(x) \partial_{t} w=0 & \text { on }(0, T) \times \Omega .\end{cases}
$$

From linear semi-group theory, we infer that

$$
w \in C^{0}\left([0, T] ; H_{0}^{1}\left(\Omega \cap B_{2 L}\right)\right) \cap C^{1}\left([0, T] ; L^{2}\left(\Omega \cap B_{2 L}\right)\right) .
$$

We set

Since

$$
v_{n}(t, x)=n\left(w\left(t+\frac{1}{n}, x\right)-w(t, x)\right) .
$$

$$
a(x) \geq \epsilon_{0}>0 \text { for }|x| \geq L
$$


and $\chi=1$ on $\{|x| \leq L\}$, therefore, $v_{n}$ is a solution of

$$
\begin{cases}\partial_{t}^{2} v_{n}-\Delta v_{n}=0 & \text { in }(0, T) \times \Omega \cap B_{2 L} \\ v_{n}=0 & \text { on }(0, T) \times \Gamma \cup\{|x|=2 L\} \\ a(x) \partial_{t} v_{n}=0 & \text { on }(0, T) \times \Omega .\end{cases}
$$

We have $\left(\omega \cap B_{2 L}, T\right)$ geometrically controls $\Omega \cap B_{2 L}$ and

$$
v_{n} \in C^{0}\left([0, T] ; H_{0}^{1}\left(\Omega \cap B_{2 L}\right)\right) \cap C^{1}\left([0, T] ; L^{2}\left(\Omega \cap B_{2 L}\right)\right),
$$

thus using the observability estimate for the wave equation in bounded domain (see e.g. 6]), we end up with

$$
E_{v_{n}}(s)=0, \text { for all } s \in[0, T]
$$

On the other hand,

$$
v_{n} \underset{n \rightarrow+\infty}{\longrightarrow} \partial_{t} w \text { in } \mathcal{D}^{\prime}((0, T) \times \Omega) .
$$

We deduce that $\partial_{t} w=0$. Recalling that $\chi=1$ on $\{|x| \leq L\}$, hence

$$
\partial_{t} z(t, x)=0 \text {, on }\{|x| \leq L\}
$$

Using $a(x) \partial_{t} z=0$ on $(0, T) \times \Omega$ along with $a(x)>\epsilon_{0}>0$ for $|x| \geq L$, we infer that $\partial_{t} z \equiv 0$ on $[0, T] \times \Omega$. This mean that $z(t, x)=z(x)$ is independent of $t$. Therefore, we have

$$
\Delta z=0 \text { and } z \in H_{D}(\Omega),
$$

we conclude from this that $z \equiv 0$ on $[0, T] \times \Omega$.

In view of the fact that the energy doesn't control the $L^{2}$ norm of the solution, we do not expect to prove an observability estimate for the global energy and this is the essential difference with the equation in a bounded domain or the Klein-Gordon equation.

We remind that under our assumptions we have the following Poincaré inequality (see [5] and [12])

$$
\|f\|_{L^{2}\left(\Omega \cap B_{R}\right)} \leq C_{R}\|\nabla f\|_{L^{2}(\Omega)} \text {, for every } f \in H_{D}(\Omega) \text { and } R \geq r_{0} .
$$

Next we show a weighted observability estimate for the local energy of solutions of the system (1.1).

Proposition 2. We assume that Hyp A holds and $\omega$ satisfies GCC. Let $\delta>0$ and $R_{0} \geq L$. Let $\varphi$ be a positive function in $C^{2}\left(\mathbb{R}_{+}\right)$such that $\varphi^{\prime}$ in $L^{\infty}\left(\mathbb{R}_{+}\right)$. We suppose that there exists a positive constant $K$ such that

$$
\sup _{\mathbb{R}_{+}}\left|\frac{\varphi^{\prime \prime}(t)}{\varphi^{\prime}(t)}\right| \leq K
$$


Moreover we assume that the function $t \longmapsto\left|\frac{\varphi^{\prime}(t)}{\varphi(t)}\right|$ is monotone decreasing and $\lim _{t \rightarrow+\infty}\left|\frac{\varphi^{\prime}(t)}{\varphi(t)}\right|=$ 0. There exist $T>0$ and $C_{T, \delta}=C\left(T, \delta, R_{0}\right)>0$, such that the following inequality

$$
\begin{aligned}
& \int_{t}^{t+T} \int_{\Omega \cap B_{R_{0}}} \varphi(q(x)+s)\left(|u|^{2}+|\nabla u|^{2}+\left|\partial_{t} u\right|^{2}\right) d x d s \\
& \leq C_{T, \delta} \int_{t}^{t+T} \int_{\Omega} a(x) \varphi(q(x)+s)\left|\partial_{t} u\right|^{2} d x d s \\
& +C_{T, \delta} \int_{t}^{t+T} \int_{\Omega} \varphi(q(x)+s)|g(s, x)|^{2} d x d s \\
& +C_{T, \delta} \int_{t}^{t+T} \int_{\Omega} \frac{\left(\varphi^{\prime}(q(x)+s)\right)^{2}}{\varphi(q(x)+s)} a(x)|u|^{2} d x d s \\
& +\delta \int_{t}^{t+T} \int_{\Omega} \varphi(q(x)+s)\left(|\nabla u|^{2}+\left|\partial_{t} u\right|^{2}\right) d x d s
\end{aligned}
$$

holds for every

$$
\text { g such that } \sqrt{\varphi} g \in L_{\text {loc }}^{2}\left(\mathbb{R}_{+}, L^{2}(\Omega)\right)
$$

for all

$$
u \in C^{0}\left(\mathbb{R}_{+}, H_{0}^{1}(\Omega)\right) \cap C^{1}\left(\mathbb{R}_{+}, L^{2}(\Omega)\right),
$$

solution of

$$
\begin{cases}\partial_{t}^{2} u-\Delta u=g & \text { in } \mathbb{R}_{+} \times \Omega, \\ u=0 & \text { on } \mathbb{R}_{+} \times \Gamma, \\ u(0, x)=u_{0} \quad \text { and } \quad \partial_{t} u(0, x)=u_{1}, & \end{cases}
$$

such that $E_{\varphi}(u)(0)<\infty$.

Proof. Let $T>0$ such that $(\omega, T)$ geometrically controls $\Omega$.

To prove this result we argue by contradiction: If (2.23) was false, there would exist a sequences $\left(t_{n}\right),\left(g_{n}\right)$ such that $\sqrt{\varphi} g_{n} \in L_{\text {loc }}^{2}\left(\mathbb{R}_{+}, L^{2}(\Omega)\right)$ and a sequence of solutions $\left(u_{n}\right)$ in $C^{0}\left(\mathbb{R}_{+}, H_{0}^{1}(\Omega)\right) \cap C^{1}\left(\mathbb{R}_{+}, L^{2}(\Omega)\right)$ with $E_{\varphi}\left(u_{n}\right)(0)<\infty$ and such that

$$
\begin{aligned}
& \int_{t_{n}}^{t_{n}+T} \int_{\Omega \cap B_{R_{0}}} \varphi(q(x)+s)\left(\left|u_{n}\right|^{2}+\left|\nabla u_{n}\right|^{2}+\left|\partial_{t} u_{n}\right|^{2}\right) d x d s \\
& \geq n\left(\int_{t_{n}}^{t_{n}+T} \int_{\Omega} a(x) \varphi(q(x)+s)\left|\partial_{t} u_{n}\right|^{2} d x d s\right) \\
& +n \int_{t_{n}}^{t_{n}+T} \int_{\Omega} \varphi(q(x)+s)\left|g_{n}(s, x)\right|^{2} d x d s \\
& +n\left(\int_{t_{n}}^{t_{n}+T} \int_{\Omega} \frac{\left(\varphi^{\prime}(q(x)+s)\right)^{2}}{\varphi(q(x)+s)} a(x)\left|u_{n}\right|^{2} d x d s\right) \\
& +\delta \int_{t_{n}}^{t_{n}+T} \int_{\Omega} \varphi(q(x)+s)\left(\left|\nabla u_{n}\right|^{2}+\left|\partial_{t} u_{n}\right|^{2}\right) d x d s .
\end{aligned}
$$

First case: The sequence $\left(t_{n}\right)$ is bounded. 
$\varphi$ is a continuous positive function on $\mathbb{R}_{+}$, therefore for all $K>R_{0}$ there exist $M>N>0$ such that

$$
N \leq \varphi\left(q(x)+t_{n}+s\right) \leq M, \text { for all }(s, x) \in[0, T] \times B_{K} .
$$

We set

$$
\begin{aligned}
& \sigma_{n}^{2}=\int_{t_{n}}^{t_{n}+T} \int_{\Omega \cap B_{R_{0}}}\left(\left|u_{n}\right|^{2}+\left|\nabla u_{n}\right|^{2}+\left|\partial_{t} u_{n}\right|^{2}\right) d x d s \\
& \text { and } v_{n}(t, x)=\frac{u_{n}\left(t_{n}+t, x\right)}{\sigma_{n}} .
\end{aligned}
$$

From (2.25) and (2.26), we infer that

$$
\begin{aligned}
& \int_{t_{n}}^{t_{n}+T} \int_{\Omega \cap B_{K}}\left(\left|\nabla v_{n}(t)\right|^{2}+\left|\partial_{t} v_{n}(t)\right|^{2}\right) d x d t \leq C_{\delta} \\
& \text { and } \int_{t_{n}}^{t_{n}+T} \int_{\Omega \cap B_{R_{0}}}\left|v_{n}(t)\right|^{2} d x d t \leq C,
\end{aligned}
$$

and

$$
\begin{aligned}
& \int_{0}^{T} \int_{\Omega \cap B_{K}} a(x)\left|\partial_{t} v_{n}(s, x)\right|^{2} d x d s \underset{n \rightarrow+\infty}{\longrightarrow} 0 \\
& \frac{1}{\sigma_{n}^{2}} \int_{0}^{T} \int_{\Omega \cap B_{K}}^{\longrightarrow}\left|g_{n}\left(s+t_{n}, x\right)\right|^{2} d x d s \underset{n \rightarrow+\infty}{\longrightarrow \rightarrow} 0,
\end{aligned}
$$

for all $K>R_{0}$. We note that since the function $t \longmapsto\left|\frac{\varphi^{\prime}(t)}{\varphi(t)}\right|$ is monotone decreasing and the the sequence $\left(t_{n}\right)$ is bounded, then for all $K \geq L$, we have

$$
\begin{aligned}
& \int_{0}^{T} \int_{\Omega \cap B_{K}} a(x)\left|v_{n}(s, x)\right|^{2} d x d s \\
& \leq C \int_{0}^{T} \int_{\Omega} \frac{\left(\varphi^{\prime}\left(q(x)+t_{n}+t\right)\right)^{2}}{\varphi\left(q(x)+t_{n}+t\right)} a(x)\left|u_{n}(s, x)\right|^{2} d x d s \underset{n \rightarrow+\infty}{\longrightarrow} 0 .
\end{aligned}
$$

Then the result above combined with (2.27), gives

$$
\int_{0}^{T} \int_{\Omega \cap B_{K}}\left(\left|v_{n}(t)\right|^{2}+\left|\nabla v_{n}(t)\right|^{2}+\left|\partial_{t} v_{n}(t)\right|^{2}\right) d x d t \leq C_{\delta} \text {, for n large enough. }
$$

We take $R_{1}$ and $R_{2}$ such that, $R_{2}>R_{1}>\max \left(R_{0}, 2 L\right)$ and let $\psi \in C_{c}^{\infty}\left(\mathbb{R}^{d}\right)$ such that $\psi=1$ on $\left\{x \in \mathbb{R}^{d}, \frac{3 L}{2} \leq|x| \leq R_{1}\right\}$ and the support of $\psi$ is contained in $\left\{x \in \mathbb{R}^{d}, L \leq|x| \leq R_{2}\right\}$. Let $0<\epsilon<<1$ and $\eta$ be a nonnegative function in $C_{c}^{\infty}(0, T)$ such that

$$
\eta(s)=1 \text { for } \epsilon \leq s \leq T-\epsilon .
$$

Now we show that

$$
\int_{\epsilon}^{T-\epsilon} \int_{\Omega \cap\left\{\frac{3 L}{2} \leq|x| \leq R_{1}\right\}}\left|\nabla v_{n}(s)\right|^{2}+\left|v_{n}(s, x)\right|^{2} d x d s \underset{n \rightarrow+\infty}{\longrightarrow} 0 .
$$

First we note that since the support of $\psi$ is contained in $\left\{L \leq|x| \leq R_{2}\right\}$ and $a(x)>\epsilon_{0}$ on $\{L \leq|x|\}$, then using (2.29) we get

$$
\epsilon_{0} \int_{0}^{T} \int_{\Omega \cap\left\{\frac{3 L}{2} \leq|x| \leq R_{1}\right\}}\left|v_{n}(s, x)\right|^{2} d x d s \leq \int_{0}^{T} \int_{\Omega \cap\left\{\frac{3 L}{2} \leq|x| \leq R_{1}\right\}} a(x)\left|v_{n}(s, x)\right|^{2} d x d s \underset{n \rightarrow+\infty}{\longrightarrow} 0 .
$$


We have, $v_{n}$ is a solution of the following system

$$
\begin{cases}\partial_{t}^{2} v_{n}-\Delta v_{n}=\frac{1}{\sigma_{n}} g_{n}(t, x) & \text { in } \mathbb{R}_{+} \times \Omega, \\ v_{n}(t, x)=0 & \text { on } \mathbb{R}_{+} \times \Gamma, \\ \left(v_{n}(0), \partial_{t} v_{n}(0)\right)=\frac{1}{\sigma_{n}}\left(u_{n}\left(t_{n}\right), \partial_{t} u_{n}\left(t_{n}\right)\right) \in H_{0}^{1}(\Omega) \times L^{2}(\Omega) . & \end{cases}
$$

We multiply Eq(2.32) by $\eta \psi^{2} v_{n}$ and integrate over $(0, T) \times \Omega$, we obtain

$$
\begin{aligned}
& \int_{0}^{T} \int_{\Omega} \eta(s) \psi^{2}(x)\left|\nabla v_{n}(s)\right|^{2} d x d s \\
= & \int_{0}^{T} \int_{\Omega} \eta^{\prime}(s) \psi^{2}(x) v_{n}(s) \partial_{t} v_{n}(s)+\eta(s) \psi^{2}(x)\left|\partial_{t} v_{n}(s)\right|^{2} d x d s \\
& +\int_{0}^{T} \int_{\Omega} \frac{1}{2} \eta(s) \Delta \psi^{2}(x)\left|v_{n}(s)\right|^{2}+\frac{1}{\sigma_{n}} \eta(s) \psi^{2}(x) g_{n}(s, x) v_{n}(s) d x d s .
\end{aligned}
$$

Using Young's inequality and the fact that $\eta$ is in $C_{c}^{\infty}(0, T)$, we infer that there exists a positive constant $c$ such that

$$
\begin{aligned}
& \int_{0}^{T} \int_{\Omega} \eta(s) \psi^{2}(x)\left|\nabla v_{n}(s)\right|^{2} d x d s \\
\leq & c \int_{0}^{T} \int_{\Omega} \psi^{2}(x)\left(\left|\partial_{t} v_{n}(s)\right|^{2}+\left|v_{n}(s)\right|^{2}\right)+\left|\Delta \psi^{2}(x)\right|\left|v_{n}(s)\right|^{2} d x d s \\
& +\frac{c}{\sigma_{n}^{2}} \int_{0}^{T} \int_{\Omega \cap B_{R_{2}}}\left|g_{n}\left(s+t_{n}, x\right)\right|^{2} d x d s,
\end{aligned}
$$

therefore

$$
\begin{aligned}
& \int_{0}^{T} \int_{\Omega} \eta(s) \psi^{2}(x)\left|\nabla v_{n}(s)\right|^{2} d x d s \\
\leq & c \int_{0}^{T} \int_{\Omega \cap B_{R_{2}}} a(x)\left|\partial_{t} v_{n}(s)\right|^{2}+a(x)\left|v_{n}(s)\right|^{2} d x d s \\
& +c \int_{0}^{T} \int_{\Omega \cap B_{R_{2}}}\left|\frac{1}{\sigma_{n}} g_{n}\left(t_{n}+s, x\right)\right|^{2} d x d s .
\end{aligned}
$$

Combining the estimate above with (2.28) and (2.29), we get

$$
\begin{aligned}
& \int_{\epsilon}^{T-\epsilon} \int_{\Omega \cap\left\{\frac{3 L}{2} \leq|x| \leq R_{1}\right\}}\left|\nabla v_{n}(s)\right|^{2} d x d s \\
& \leq \int_{0}^{T} \int_{\Omega} \eta(s) \psi^{2}(x)\left|\nabla v_{n}(s)\right|^{2} d x d s \underset{n \rightarrow+\infty}{\longrightarrow} 0,
\end{aligned}
$$

we note that in the inequality above we have used the fact that $\psi=1$ on $\left\{x \in \mathbb{R}^{d}, \frac{3 L}{2} \leq|x| \leq R_{1}\right\}$ and $\eta=1$ on $[\epsilon, T-\epsilon]$.

Let $\chi \in C_{c}^{\infty}\left(\mathbb{R}^{d}\right)$ such that $\chi=1$ on $\{|x| \leq R\}$ and the support of $\chi$ is contained in $\left\{|x| \leq R_{1}\right\}$ with $R_{1}>R>\max \left(R_{0}, 2 L\right)$. We set $W_{n}=\chi v_{n}$, then $W_{n}$ is a solution of the following system

$$
\begin{cases}\partial_{t}^{2} W_{n}-\Delta W_{n}=-2 \nabla \chi \nabla v_{n}-v_{n} \Delta \chi+\frac{1}{\sigma_{n}} \chi g_{n}(t, x) & \mathbb{R}_{+} \times \Omega \cap B_{R_{1}}, \\ W_{n}=0 & \mathbb{R}_{+} \times \Gamma \cup\left\{|x|=R_{1}\right\} \\ \left(W_{n}(0), \partial_{t} W_{n}(0)\right)=\chi\left(v_{n}(0), \partial_{t} v_{n}(0)\right) . & \end{cases}
$$


In addition we have

$$
W_{n} \in C\left((0, T), H_{0}^{1}\left(\Omega \cap B_{R_{1}}\right)\right) \cap C^{1}\left((0, T), L^{2}\left(\Omega \cap B_{R_{1}}\right)\right) .
$$

Now we show that

$$
\sup _{[0, T]} E_{W_{n}}(s) \leq C_{T, \delta}, \text { for n large enough. }
$$

First we note that we have the following energy identity

$$
t E_{W_{n}}(t)=\int_{0}^{t} E_{W_{n}}(s) d s+\int_{0}^{t} \int_{\Omega} s\left(-2 \nabla \chi \nabla v_{n}-v_{n} \Delta \chi+\frac{1}{\sigma_{n}} \chi g_{n}\right) \partial_{t} W_{n} d x d s
$$

for all $0 \leq t \leq T$. Then using Young's inequality and the fact that the support of $W_{n}$ is contained in $\left\{|x| \leq R_{1}\right\}$, we deduce that

$E_{W_{n}}(T)$

$$
\begin{aligned}
& \leq \frac{c}{T}\left(\int_{0}^{T}\left(E_{W_{n}}(s)+T \int_{\Omega \cap B_{R_{1}}}\left|-2 \nabla \chi \nabla v_{n}-v_{n} \Delta \chi+\frac{1}{\sigma_{n}} \chi g_{n}\right|^{2}+\left|\partial_{t} W_{n}\right|^{2} d x\right) d s\right) \\
& \leq \frac{c}{T} \int_{0}^{T} \int_{\Omega \cap B_{R_{1}}}\left|\nabla v_{n}\right|^{2}+\left|\partial_{t} v_{n}\right|^{2}+\left|v_{n}\right|^{2}+\left|\frac{1}{\sigma_{n}} \chi g_{n}\right|^{2} d x d s .
\end{aligned}
$$

Combining the estimate above with (2.28) and (2.30), we obtain

$$
E_{W_{n}}(T) \leq C_{T, \delta}, \text { for n large enough. }
$$

On the other hand, we have the following energy identity

$$
E_{W_{n}}(t)=E_{W_{n}}(T)+\int_{t}^{T} \int_{\Omega \cap B_{R_{1}}}\left(-2 \nabla \chi \nabla v_{n}-v_{n} \Delta \chi+\frac{1}{\sigma_{n}} \chi g_{n}\right) \partial_{t} W_{n} d x d s
$$

for all $0 \leq t \leq T$. Using Young's inequality and making some arrangement, we deduce that

$$
\begin{aligned}
& E_{W_{n}}(t) \\
& \leq E_{W_{n}}(T)+c \int_{0}^{T} \int_{\Omega \cap B_{R_{1}}}\left|-2 \nabla \chi \nabla v_{n}-v_{n} \Delta \chi+\frac{1}{\sigma_{n}} \chi g_{n}\right|^{2}+\left|\partial_{t} v_{n}\right|^{2} d x d s,
\end{aligned}
$$

for all $0 \leq t \leq T$. The estimate above combined with (2.28), (2.30) and (2.34) gives (2.33) .

The next step is to show that

$$
\int_{0}^{T} E_{W_{n}}(s) d s \underset{n \rightarrow+\infty}{\longrightarrow} 0 .
$$

For $\epsilon$ small enough, we have $\left(\omega \cap B_{R_{1}}, T-2 \epsilon\right)$ geometrically controls $\Omega \cap B_{R_{1}}$. Therefore, using the control theory of the wave equation in bounded domain, we deduce that the following observability estimate holds

$E_{W_{n}}(\epsilon)$

$\leq C_{\epsilon, T}\left(\int_{\epsilon}^{T-\epsilon} \int_{\Omega \cap B_{R_{1}}} a(x)\left|\partial_{t} v_{n}\right|^{2}+\left|-2 \nabla \chi \nabla v_{n}-v_{n} \Delta \chi+\frac{1}{\sigma_{n}} \chi g_{n}\right|^{2} d x d s\right)$,

(we can show this result using [6].) Recalling

$$
\nabla \chi=0 \text { on }\{|x| \leq 2 L\} \text { and Supp } \chi \subset\left\{|x| \leq R_{1}\right\} .
$$


Hence (2.31) and (2.28) give

$$
\int_{\epsilon}^{T-\epsilon} \int_{\Omega \cap B_{R_{1}}}\left|-2 \nabla \chi \nabla v_{n}-v_{n} \Delta \chi+\frac{1}{\sigma_{n}} \chi g_{n}\right|^{2} d x d s \underset{n \rightarrow+\infty}{\longrightarrow} 0 .
$$

Combining the estimate above with (2.28), we get

$$
E_{W_{n}}(\epsilon) \underset{n \rightarrow+\infty}{\longrightarrow} 0,
$$

for all $\epsilon>0$ small enough, such that $\left(\omega \cap B_{R_{1}}, T-2 \epsilon\right)$ geometrically controls $\Omega \cap$ $B_{R_{1}}$. On the other hand the energy estimate for the nonhomogeneous wave equation, gives

$$
\begin{aligned}
& E_{W_{n}}(s) \\
& \leq 2 e^{T}\left(E_{W_{n}}(\epsilon)+\int_{\epsilon}^{T-\epsilon} \int_{\Omega \cap B_{R_{1}}}\left|-2 \nabla \chi \nabla v_{n}-v_{n} \Delta \chi+\frac{1}{\sigma_{n}} \chi g_{n}\right|^{2} d x d t\right),
\end{aligned}
$$

for $\epsilon \leq s \leq T-\epsilon$. Using (2.37) and (2.38), we see that

$E_{W_{n}}(s)$

$\leq C\left(E_{W_{n}}(\epsilon)+\int_{\epsilon}^{T-\epsilon} \int_{\Omega \cap B_{R_{1}}}\left|-2 \nabla \chi \nabla v_{n}-v_{n} \Delta \chi+\frac{1}{\sigma_{n}} \chi g_{n}\right|^{2} d x d t\right) \underset{n \rightarrow+\infty}{\longrightarrow} 0$,

for all $s \in[\epsilon, T-\epsilon]$. We conclude that

$$
E_{W_{n}}(s) \underset{n \rightarrow+\infty}{\longrightarrow} 0, \text { for all } 0<s<T \text {. }
$$

Using (2.33) and applying the dominated convergence theorem, we obtain (2.35) .

Now (2.35) and the fact that $\chi=1$ on $\{|x| \leq R\}$ along with (2.29), give

$$
\int_{0}^{T} \int_{\Omega \cap B_{R}}\left|\nabla v_{n}(s)\right|^{2}+\left|\partial_{t} v_{n}(s)\right|^{2} d x d s \underset{n \rightarrow+\infty}{\longrightarrow} 0 .
$$

On the other hand let $\theta \in C_{c}^{\infty}\left(\mathbb{R}^{d}\right)$ such that $\theta=1$ on $\left\{|x| \leq R_{0}\right\}$ and the support of $\theta$ is contained in $\{|x| \leq R\}$. Using Poincaré's inequality, we obtain

$$
\begin{aligned}
& \int_{0}^{T} \int_{\Omega \cap B_{R_{0}}}\left|v_{n}(s, x)\right|^{2} d x d s \\
\leq & C \int_{0}^{T} \int_{\Omega \cap B_{R}}\left|v_{n}(s, x) \nabla \theta(x)\right|^{2}+\left|\theta(x) \nabla v_{n}(s, x)\right|^{2} d x d s .
\end{aligned}
$$

The estimate above combined with (2.41) and (2.29), give

$$
\int_{0}^{T} \int_{\Omega \cap B_{R_{0}}}\left|v_{n}(s)\right|^{2}+\left|\nabla v_{n}(s)\right|^{2}+\left|\partial_{t} v_{n}(s)\right|^{2} d x d s \underset{n \rightarrow+\infty}{\longrightarrow} 0 .
$$

The contradiction follows from the fact that

$$
\begin{aligned}
1 & =\frac{1}{\sigma_{n}^{2}} \int_{t_{n}}^{t_{n}+T} \int_{\Omega \cap B_{R_{0}}} \varphi\left(s+t_{n}+q(x)\right)\left(\left|u_{n}\right|^{2}+\left|\nabla u_{n}\right|^{2}+\left|\partial_{t} u_{n}\right|^{2}\right) d x d s \\
& \leq C \int_{0}^{T} \int_{\Omega \cap B_{R_{0}}}\left(\left|v_{n}\right|^{2}+\left|\nabla v_{n}\right|^{2}+\left|\partial_{t} v_{n}\right|^{2}\right) d x d s \underset{n \rightarrow+\infty}{\longrightarrow} 0 .
\end{aligned}
$$


Second case : The sequence $t_{n} \underset{n \rightarrow+\infty}{\longrightarrow}+\infty$. We set

$$
\begin{aligned}
& \sigma_{n}^{2}=\int_{t_{n}}^{t_{n}+T} \int_{\Omega \cap B_{R_{0}}} \varphi(q(x)+s)\left(\left|u_{n}\right|^{2}+\left|\nabla u_{n}\right|^{2}+\left|\partial_{t} u_{n}\right|^{2}\right) d x d s \\
& \text { and } v_{n}(t, x)=\frac{\left(\varphi\left(q(x)+t_{n}+t\right)\right)^{\frac{1}{2}} u_{n}\left(t_{n}+t, x\right)}{\sigma_{n}} .
\end{aligned}
$$

From (2.25), we infer that

$$
\begin{aligned}
& \frac{1}{\sigma_{n}^{2}} \int_{t_{n}}^{t_{n}+T} \int_{\Omega} \varphi(q(x)+t)\left(\left|\nabla u_{n}(t)\right|^{2}+\left|\partial_{t} u_{n}(t)\right|^{2}\right) d x d t \leq \frac{1}{\delta} \\
& \text { and } \int_{t_{n}}^{t_{n}+T} \int_{\Omega \cap B_{R_{0}}}\left|v_{n}(t)\right|^{2} d x d t \leq 1,
\end{aligned}
$$

and

$$
\begin{aligned}
& \frac{1}{\sigma_{n}^{2}} \int_{t_{n}}^{t_{n}+T} \int_{\Omega} a(x) \varphi(q(x)+s)\left|\partial_{t} u_{n}\right|^{2} d x d s \underset{n \rightarrow+\infty}{\longrightarrow} 0 \\
& \frac{1}{\sigma_{n}^{2}} \int_{t_{n}}^{t_{n}+T} \int_{\Omega}^{\longrightarrow} \varphi(q(x)+s)\left|g_{n}(s, x)\right|^{2} d x d s \underset{n \rightarrow+\infty}{\longrightarrow} 0 \\
& \frac{1}{\sigma_{n}^{2}} \int_{t_{n}}^{t_{n}+T} \int_{\Omega}^{\longrightarrow} \frac{\left(\varphi^{\prime}(q(x)+s)\right)^{2}}{\varphi(q(x)+s)} a(x)\left|u_{n}\right|^{2} d x d s \underset{n \rightarrow+\infty}{\longrightarrow} 0 .
\end{aligned}
$$

It is clear that $v_{n}$ is a solution of the following system

$$
\begin{cases}\partial_{t}^{2} v_{n}-\Delta v_{n}=f_{n}(t, x) & \text { in } \mathbb{R}_{+} \times \Omega \\ v_{n}(t, x)=0 & \text { on } \mathbb{R}_{+} \times \Gamma \\ \left(v_{n}(0), \partial_{t} v_{n}(0)\right) \in H_{0}^{1}(\Omega) \times L^{2}(\Omega), & \end{cases}
$$

where

$$
\begin{aligned}
& f_{n}(t, x)=\frac{1}{2 \sigma_{n}}\left[\left(\varphi^{\prime \prime}(\varphi)^{-\frac{1}{2}}-\frac{1}{2}\left(\varphi^{\prime}\right)^{2} \varphi^{-3 / 2}\right) \frac{|x|^{2}}{q^{2}}\right] u_{n}\left(t_{n}+t\right) \\
& +\frac{1}{2 \sigma_{n}}\left[\left(\frac{d}{q}-\frac{|x|^{2}}{q^{3}}\right) \varphi^{\prime}(\varphi)^{-\frac{1}{2}}\right] u_{n}\left(t_{n}+t\right) \\
& +\frac{1}{2 \sigma_{n}}\left[\varphi^{\prime \prime}(\varphi)^{-\frac{1}{2}}-\frac{1}{2}\left(\varphi^{\prime}\right)^{2} \varphi^{-3 / 2}\right] u_{n}\left(t_{n}+t\right)-\frac{1}{\sigma_{n}} \varphi^{\frac{1}{2}} g_{n}\left(t_{n}+t, x\right) \\
& +\frac{\varphi^{\prime}(\varphi)^{-\frac{1}{2}}}{\sigma_{n}}\left(\partial_{t} u_{n}\left(t_{n}+t\right)+\frac{x \cdot \nabla u_{n}\left(t_{n}+t\right)}{q}\right),
\end{aligned}
$$

where $\varphi^{(j)}(t, x)=\varphi^{(j)}\left(q(x)+t+t_{n}\right)$, for $j=0,1,2$.

Now we will show that

$$
\int_{0}^{T} \int_{\Omega}\left|f_{n}(s, x)\right|^{2} d x d s \underset{n \rightarrow+\infty}{\longrightarrow} 0
$$


Using (2.43) and the fact that $\lim _{t \rightarrow+\infty}\left|\frac{\varphi^{\prime}(t)}{\varphi(t)}\right|=0$, we obtain

$$
\begin{aligned}
& \int_{0}^{T} \int_{\Omega}\left|\frac{1}{2 \sigma_{n}}\left[\left(\varphi^{\prime \prime}(\varphi)^{-\frac{1}{2}}-\frac{1}{2}\left(\varphi^{\prime}\right)^{2} \varphi^{-3 / 2}\right) \frac{|x|^{2}}{q^{2}}\right] u_{n}\left(t_{n}+t\right)\right|^{2} d x d t \\
& +\int_{0}^{T} \int_{\Omega}\left|\frac{1}{2 \sigma_{n}}\left[\left(\frac{d}{q}-\frac{|x|^{2}}{q^{3}}\right) \varphi^{\prime}(\varphi)^{-\frac{1}{2}}\right] u_{n}\left(t_{n}+t\right)\right|^{2} d x d t \\
& +\int_{0}^{T} \int_{\Omega}\left|\frac{1}{2 \sigma_{n}}\left[\varphi^{\prime \prime}(\varphi)^{-\frac{1}{2}}-\frac{1}{2}\left(\varphi^{\prime}\right)^{2} \varphi^{-3 / 2}\right] u_{n}\left(t_{n}+t\right)\right|^{2} d x d t \\
& \leq \frac{C}{\sigma_{n}^{2}} \int_{t_{n}}^{t_{n}+T} \int_{\Omega} \frac{\left(\varphi^{\prime}(q(x)+s)\right)^{2}}{\varphi(q(x)+s)}\left(1+\left(\frac{\varphi^{\prime}\left(t_{n}\right)}{\varphi\left(t_{n}\right)}\right)^{2}\right)\left|u_{n}\right|^{2} d x d s \\
& \leq \frac{C}{\sigma_{n}^{2}}\left(\frac{\varphi^{\prime}\left(t_{n}\right)}{\varphi\left(t_{n}\right)}\right)^{2} \int_{t_{n}}^{t_{n}+T} \int_{\Omega \cap B_{L}} \varphi(q(x)+s)\left|u_{n}\right|^{2} d x d s \\
& +\frac{C}{\epsilon_{0} \sigma_{n}^{2}} \int_{t_{n}}^{t_{n}+T} \int_{\Omega}^{\frac{\left(\varphi^{\prime}(q(x)+s)\right)^{2}}{\varphi(q(x)+s)}} a(x)\left|u_{n}\right|^{2} d x d s \\
& \leq C\left(\frac{\varphi^{\prime}\left(t_{n}\right)}{\varphi\left(t_{n}\right)}\right)^{2}+\frac{C}{\epsilon_{0} \sigma_{n}^{2}} \int_{t_{n}}^{t_{n}+T} \int_{\Omega} \frac{\left(\varphi^{\prime}(q(x)+s)\right)^{2}}{\varphi(q(x)+s)} a(x)\left|u_{n}\right|^{2} d x d s \underset{n \rightarrow+\infty}{\longrightarrow} 0 .
\end{aligned}
$$

Now we estimate the remaining term of $f_{n}$. Turn into account of (2.42), we get,

$$
\begin{aligned}
& \int_{0}^{T} \int_{\Omega}\left|\frac{\varphi^{\prime}(\varphi)^{-\frac{1}{2}}}{\sigma_{n}}\left(\partial_{t} u_{n}\left(t_{n}+t\right)+\frac{x \cdot \nabla u_{n}\left(t_{n}+t\right)}{q}\right)\right|^{2} d x d t \\
& \leq \frac{C}{\sigma_{n}^{2}}\left(\frac{\varphi^{\prime}\left(t_{n}\right)}{\varphi\left(t_{n}\right)}\right)^{2} \int_{0}^{T} \int_{\Omega} \varphi\left(q(x)+\left(t_{n}+t\right)\right)\left(\left|\partial_{t} u_{n}\left(t_{n}+t\right)\right|^{2}+\left|\nabla u_{n}\left(t_{n}+t\right)\right|^{2}\right) d x d t \\
& \leq \frac{C}{\delta}\left(\frac{\varphi^{\prime}\left(t_{n}\right)}{\varphi\left(t_{n}\right)}\right)^{2} \underset{n \rightarrow+\infty}{\longrightarrow} 0 .
\end{aligned}
$$

The results above combined with (2.43), gives (2.44) .

The next step is to show the boundeness of the energy of $v_{n}$. It is easy to see that

$$
\begin{aligned}
& \int_{0}^{T} E_{v_{n}}(t) d t \leq \frac{c}{\sigma_{n}^{2}} \int_{t_{n}}^{t_{n}+T} \int_{\Omega} \varphi(q(x)+t)\left(\left|\nabla u_{n}(t)\right|^{2}+\left|\partial_{t} u_{n}(t)\right|^{2}\right) d x d t \\
& +\frac{c}{\sigma_{n}^{2}} \int_{t_{n}}^{t_{n}+T} \int_{\Omega} \frac{\left(\varphi^{\prime}(q(x)+t)\right)^{2}}{\varphi(q(x)+t)}\left|u_{n}(t)\right|^{2} d x d t .
\end{aligned}
$$

Now using (2.42) and (2.43) we infer that there exists a positive constant $C_{\delta}$ such that

$$
\int_{0}^{T} E_{v_{n}}(t) d t \leq C_{\delta}, \text { for n large enough. }
$$

On the other hand, we have

$$
E_{v_{n}}(t) \leq \frac{c}{t}\left(\int_{0}^{T}\left(E_{v_{n}}(s)+s \int_{\Omega}\left|f_{n}(s, x)\right|^{2} d x\right) d s\right),
$$

for all $0<t \leq T$. Turn into account of the estimate above along with (2.45) and (2.44), we obtain

$$
E_{v_{n}}(T) \leq C_{T, \delta}, \text { for n large enough. }
$$


On the other hand, from the energy identity, we see that

$$
E_{v_{n}}(t) \leq E_{v_{n}}(T)+\int_{0}^{T}\left(E_{v_{n}}(s)+\int_{\Omega}\left|f_{n}(s, x)\right|^{2} d x\right) d s
$$

for all $0 \leq t \leq T$. The estimate above combined with (2.45) and (2.46) gives

$$
\sup _{[0, T]} E_{v_{n}}(s) \leq C_{T, \delta}, \text { for n large enough. }
$$

The last step is to show that

$$
\int_{0}^{T} \int_{\Omega} a(x)\left|\partial_{t} v_{n}\right|^{2} d x d t \underset{n \rightarrow+\infty}{\longrightarrow} 0 .
$$

We have

$$
\begin{aligned}
& \int_{0}^{T} \int_{\Omega} a(x)\left|\partial_{t} v_{n}\right|^{2} d x d t \leq \frac{2}{\sigma_{n}^{2}} \int_{t_{n}}^{t_{n}+T} \int_{\Omega} \frac{\left(\varphi^{\prime}(q(x)+s)\right)^{2}}{\varphi(q(x)+s)} a(x)\left|u_{n}(s)\right|^{2} d x d s \\
& +\frac{2}{\sigma_{n}^{2}} \int_{t_{n}}^{t_{n}+T} \int_{\Omega} \varphi(q(x)+s) a(x)\left|\partial_{t} u_{n}\right|^{2} d x d s .
\end{aligned}
$$

Using (2.43), we get (2.48) .

For the rest of the proof we have only to argue as in [7, Proof of proposition 2].

\section{Proof of Theorem 1}

3.1. Preliminary results. Throughout this section we use the following notations:

Let $\beta$ be a real number such that

$$
\begin{array}{ll}
\beta>-1 & \text { if } 1<r<1+\frac{2}{d} \\
-1<\beta<\frac{3-r}{r-1} & \text { if } r=1+\frac{2}{d} .
\end{array}
$$

Let $\psi \in C_{0}^{\infty}\left(\mathbb{R}^{d}\right)$ such that $0 \leq \psi \leq 1$ and

$$
\psi(x)= \begin{cases}1 & \text { for }|x| \leq L \\ 0 & \text { for }|x| \geq 2 L\end{cases}
$$

Finally we set

$$
\begin{aligned}
& \varphi(s)=\ln ^{\beta+1}(b+s), f(s)=\frac{\ln ^{\beta}(b+s)}{b+s}, f_{1}(s)=\frac{\ln ^{\beta}(b+s)}{(b+s)^{2}} \\
& \text { and } f_{2}(s)=\frac{\ln ^{\beta-r+1}(b+s)}{(b+s)^{r}},
\end{aligned}
$$

with

$$
\ln b=\max \left((2(r+1))^{r+1}, \frac{\beta+1-r}{r-1},(8(r+1)(\beta+1))^{r+1}\right) .
$$

Proposition 3. We assume that Hyp $A$ holds and $(\omega, T)$ geometrically controls $\Omega$. Let $\beta>-1$. Let $\delta>0$ and $R_{0}>L$. There exists $C_{T, \delta}=C\left(T, \delta, R_{0}\right)>0$, such that the following 
inequality

$$
\begin{aligned}
& \int_{t}^{t+T} \int_{\Omega \cap B_{R_{0}}} f(q(x)+s)\left(|u|^{2}+|\nabla u|^{2}+\left|\partial_{t} u\right|^{2}\right) d x d s \\
& \leq C_{T, \delta} \int_{t}^{t+T} \int_{\Omega} a(x) f(q(x)+s)\left(\left|\partial_{t} u\right|^{2}+\left|\partial_{t} u\right|^{2 r}\right) d x d s \\
& +C_{T, \delta} \int_{t}^{t+T} \int_{\Omega} a(x) f_{1}^{\prime}(q(x)+s)|u|^{2} d x d s \\
& +\delta \int_{t}^{t+T} \int_{\Omega} f(q(x)+s)\left(|\nabla u(s)|^{2}+\left|\partial_{t} u(s)\right|^{2}\right) d x d s,
\end{aligned}
$$

holds for every $t \geq 0$ and for all $u$ solution of (1.1) with initial data $\left(u_{0}, u_{1}\right)$ in $H_{0}^{1}(\Omega) \cap$ $H^{2}(\Omega) \times H_{0}^{1}(\Omega)$.

Proof. In view of $f \in L^{\infty}\left(\mathbb{R}_{+}\right)$, we have $E_{f}(u)(0)<\infty$. On the other hand, it is clear that $f^{\prime} \in L^{\infty}\left(\mathbb{R}_{+}\right)$and there exists a positive constant $K$, such that

$$
\sup _{\mathbb{R}_{+}}\left|\frac{f^{\prime \prime}(t)}{f^{\prime}(t)}\right| \leq K
$$

In addition the function $t \longmapsto\left|\frac{f^{\prime}(t)}{f(t)}\right|$ is decreasing and $\lim _{t \rightarrow+\infty}\left|\frac{f^{\prime}(t)}{f(t)}\right|=0$. Moreover there exists $C>0$, such that

$$
\frac{\left(f^{\prime}(t)\right)^{2}}{f(t)} \leq C\left(-f_{1}^{\prime}(t)\right), \text { for all } t \geq 0
$$

Since

$$
\partial_{t} u \in L^{\infty}\left(\mathbb{R}_{+}, H_{0}^{1}(\Omega)\right),
$$

therefore, from Sobolev imbedding, we deduce that

$$
\sqrt{a(x) f(q(x)+s)}\left|\partial_{t} u\right|^{r} \in L_{l o c}^{2}\left(\mathbb{R}_{+}, L^{2}(\Omega)\right) .
$$

By taking into account of the results above, we can use proposition 2 and we obtain (3.1). This finishes the proof of the proposition.

In order to prove theorem 1 we need the following result.

Lemma 2. Let $T>0$ and $u$ be the solution of (1.1) with initial data in $H_{0}^{1}(\Omega) \cap H^{2}(\Omega) \times$ $H_{0}^{1}(\Omega)$ such that

$$
E_{\varphi}(u)(0)=\int_{\Omega} \varphi(q(x))\left(\left|\nabla u_{0}\right|^{2}+\left|u_{1}\right|^{2}\right) d x<\infty .
$$

We set $\chi=1-\psi$ and

$$
\begin{aligned}
& X(t)=\int_{\Omega} f(q(x)+t) \chi^{2}(x) u(t) \partial_{t} u(t) d x+\frac{k_{1}}{2} \int_{\Omega} a(x) f_{1}(q(x)+t)|u(t)|^{2} d x \\
& +\int_{\Omega} a(x) f_{2}(q(x)+t)|u(t)|^{r+1} d x+\frac{k}{2} \int_{\Omega} \ln ^{\beta+1}(b+q(x)+t)\left(|\nabla u|^{2}+\left|\partial_{t} u\right|^{2}\right) d x,
\end{aligned}
$$

where

$$
k=\frac{1}{4(\beta+1)}, k_{1}>0
$$


We have

$$
\begin{aligned}
& X(t+T)-X(t)+\frac{1}{4} \int_{t}^{t+T} \int_{\Omega} f(q(x)+s)\left(|\nabla u|^{2}+\left|\partial_{t} u\right|^{2}\right) d x d s \\
& -\left(\frac{k_{1}}{4}-\frac{2(1+|\beta|)}{\epsilon_{0}}\right) \int_{t}^{t+T} \int_{\Omega} a(x) f_{1}^{\prime}(q(x)+s)|u|^{2} d x d s \\
& -\frac{1}{2} \int_{t}^{t+T} \int_{\Omega} a(x) f_{2}^{\prime}(q(x)+s)|u|^{r+1} d x d s \\
& +\frac{1}{8(\beta+1)} \int_{t}^{t+T} \int_{\Omega} a(x) \ln ^{\beta+1}(b+q(x)+s)\left|\partial_{t} u\right|^{r+1} d x d s \\
& \leq\left(3+\frac{1}{2}\left\|\nabla \chi^{2}\right\|_{\infty}\right) \int_{t}^{t+T} \int_{\Omega \cap B_{2 L}} f(q(x)+s)\left(|u|^{2}+|\nabla u|^{2}+\left|\partial_{t} u\right|^{2}\right) d x d s \\
& +2\left(\frac{1}{\epsilon_{0}}+\frac{4(1+|\beta|)}{\epsilon_{0}^{2} k_{1}}+4 k_{1}\right) \int_{t}^{t+T} \int_{\Omega} a(x) f(q(x)+t)\left|\partial_{t} u\right|^{2} d x d s .
\end{aligned}
$$

Proof. First (3.2) allows us to apply (2.3) and to obtain

$$
\begin{aligned}
& E_{\varphi}(u)(t+T)+\int_{t}^{t+T} \int_{\Omega} a(x) \varphi(q(x)+s)\left|\partial_{t} u\right|^{r+1} d x d s \\
& \leq E_{\varphi}(u)(t)+(\beta+1) \int_{t}^{t+T} \int_{\Omega} f(q(x)+s)\left(|\nabla u|^{2}+\left|\partial_{t} u\right|^{2}\right) d x d s .
\end{aligned}
$$

We set

$$
\begin{aligned}
& X_{0}(t)=\int_{\Omega} f(q(x)+t) \chi^{2}(x) u(t) \partial_{t} u(t) d x+\frac{k_{1}}{2} \int_{\Omega} a(x) f_{1}(q(x)+t)|u(t)|^{2} d x \\
& +\int_{\Omega} a(x) f_{2}(q(x)+t)|u(t)|^{r+1} d x .
\end{aligned}
$$

Therefore, we have

$$
\begin{aligned}
& \frac{d}{d t} X_{0}(t)=\int_{\Omega}\left(\left|\partial_{t} u(t)\right|^{2}-|\nabla u(t)|^{2}-a(x)\left|\partial_{t} u(t)\right|^{r-1} u \partial_{t} u(t)\right) \chi^{2}(x) f(q(x)+t) d x \\
& -\int_{\Omega} \chi^{2}(x) f^{\prime}(q(x)+t) u(t) \frac{x \cdot \nabla u(t)}{q(x)}+f(q(x)+t) \nabla \chi^{2}(x) \nabla u(t) d x \\
& +\int_{\Omega} f^{\prime}(q(x)+t) \chi^{2}(x) u(t) \partial_{t} u(t) d x \\
& +k_{1}\left(\int_{\Omega} a(x) f_{1}(q(x)+t) u(t) \partial_{t} u(t) d x+\frac{1}{2} \int_{\Omega} a(x) f_{1}^{\prime}(q(x)+t)|u(t)|^{2} d x\right) \\
& +\int_{\Omega} a(x) f_{2}^{\prime}(q(x)+t)|u|^{r+1} d x+(r+1) \int_{\Omega} a(x) f_{2}(q(x)+t)|u|^{r-1} u \partial_{t} u d x .
\end{aligned}
$$

A direct computation gives

$$
\begin{aligned}
& \frac{\left(f^{\prime}(s)\right)^{2}}{f(s)} \leq(1+|\beta|) \frac{\ln ^{\beta}(b+s)}{(b+s)^{3}} \leq-(1+|\beta|) f_{1}^{\prime}(s) \\
& \text { and } \\
& \frac{\left(f_{1}(s)\right)^{2}}{f(s)}=\frac{\ln ^{\beta}(b+s)}{(b+s)^{3}} \leq-f_{1}^{\prime}(s) .
\end{aligned}
$$


We note that $\|\chi\|_{\infty} \leq 1$. Using Young's inequality and the fact that the support of $\chi$ is contained in $\{|x| \geq L\}$ and

$$
a(x)>\epsilon_{0}>0 \text { for }|x| \geq L
$$

we deduce that

$$
\begin{aligned}
& \left|\int_{\Omega} f^{\prime}(q(x)+t) \chi^{2}(x) u(t) \partial_{t} u(t) d x\right| \\
& \leq-\frac{k_{1}}{8} \int_{\Omega} a(x) f_{1}^{\prime}(q(x)+t)|u(t)|^{2} d x+\frac{8(1+|\beta|)}{\epsilon_{0}^{2} k_{1}} \int_{\Omega} a(x) f(q(x)+t)\left|\partial_{t} u(t)\right|^{2} d x,
\end{aligned}
$$

and

$$
\begin{aligned}
& \left|k_{1} \int_{\Omega} a(x) f_{1}(q(x)+t) u(t) \partial_{t} u(t) d x\right| \\
& \leq-\frac{k_{1}}{8} \int_{\Omega} a(x) f_{1}^{\prime}(q(x)+t)|u(t)|^{2} d x+8 k_{1} \int_{\Omega} a(x) f(q(x)+t)\left|\partial_{t} u(t)\right|^{2} d x .
\end{aligned}
$$

Using the same arguments we also deduce that

$$
\begin{aligned}
& \int_{\Omega} \chi^{2}(x) f^{\prime}(q(x)+t) u(t) \frac{x \cdot \nabla u(t)}{q(x)} d x \\
& \leq \frac{1}{2} \int_{\Omega} f(q(x)+t)|\nabla u(t)|^{2} d x-\frac{2(1+|\beta|)}{\epsilon_{0}} \int_{\Omega} a(x) f_{1}^{\prime}(q(x)+t)|u(t)|^{2} d x .
\end{aligned}
$$

Since the support of $\psi$ is contained in $\{|x| \leq 2 L\}$ and

$$
a(x)>\epsilon_{0} \text { for }|x| \geq L,
$$

therefore we see that

$$
\begin{aligned}
& \int_{\Omega}\left(\left|\partial_{t} u(t)\right|^{2}-|\nabla u(t)|^{2}\right) \chi^{2}(x) f(q(x)+t) d x \\
& =\int_{\Omega} f(q(x)+t)\left(1-2 \psi(x)+\psi^{2}(x)\right)\left(\left|\partial_{t} u(t)\right|^{2}-|\nabla u(t)|^{2}\right) d x \\
& \leq \frac{2}{\epsilon_{0}} \int_{\Omega} a(x) f(q(x)+t)\left|\partial_{t} u(t)\right|^{2} d x \\
& -\int_{\Omega} f(q(x)+t)\left(\left|\partial_{t} u(t)\right|^{2}+|\nabla u(t)|^{2}\right) d x \\
& +3 \int_{\Omega \cap B_{2 L}} f(q(x)+t)\left(\left|\partial_{t} u(t)\right|^{2}+|\nabla u(t)|^{2}\right) d x .
\end{aligned}
$$

We note that the support of $\nabla \chi^{2}$ is contained in $\{|x| \leq 2 L\}$, using Young's inequality, we deduce that

$$
\begin{aligned}
& \left|-\int_{\Omega} f(q(x)+t) u(t) \nabla \chi^{2}(x) \nabla u(t) d x\right| \\
& \leq \frac{1}{2}\left\|\nabla \chi^{2}\right\|_{\infty} \int_{\Omega \cap B_{2 L}} f(q(x)+t)\left(|u(t)|^{2}+|\nabla u(t)|^{2}\right) d x .
\end{aligned}
$$

Since

$$
\ln b \geq \frac{\beta+1-r}{r-1}
$$


therefore a direct computation gives

$$
\begin{aligned}
& -f_{2}(s) \geq \frac{\ln ^{\beta-r+1}(b+s)}{(b+s)^{r+1}} \\
& (f(s))^{r+1} \ln ^{-r(\beta+1)}(b+s) \leq \frac{-f_{2}^{\prime}(s)}{\ln (b+s)} \\
& \left(f_{2}(s)\right)^{\frac{r+1}{r}} \ln ^{-\frac{\beta+1}{r}}(b+s) \leq \frac{-f_{2}^{\prime}(s)}{\ln (b+s)}
\end{aligned}
$$

Now we can estimate the last term of the RHS of (3.5) . Hölder's inequality along with Young's inequality, leads to

$$
\begin{aligned}
& \int_{\Omega} a(x) f(q(x)+s)\left|\partial_{t} u(t)\right|^{r-1} u \partial_{t} u d x \\
& \leq(\ln b)^{-\frac{1}{r+1}}\left(\int_{\Omega} a(x) \ln ^{\beta+1}(b+q(x)+s)\left|\partial_{t} u\right|^{r+1} d x\right)^{\frac{r}{r+1}}\left(-\int_{\Omega} a(x) f_{2}^{\prime}(q(x)+s)|u|^{r+1} d x\right)^{\frac{1}{r+1}} \\
& \leq(\ln b)^{-\frac{1}{r+1}} \int_{\Omega} a(x) \ln ^{\beta+1}(b+q(x)+s)\left|\partial_{t} u\right|^{r+1} d x-(\ln b)^{-\frac{1}{r+1}} \int_{\Omega} a(x) f_{2}^{\prime}(q(x)+s)|u|^{r+1} d x
\end{aligned}
$$

and

$$
\begin{aligned}
& (r+1) \int_{\Omega} a(x) f_{2}(q(x)+s)|u|^{r-1} u \partial_{t} u d x \\
& \leq(r+1)(\ln b)^{-\frac{r}{r+1}}\left(\int_{\Omega} a(x) \ln ^{\beta+1}(b+q(x)+s)\left|\partial_{t} u\right|^{r+1} d x\right)^{\frac{1}{r+1}} \\
& \times\left(-\int_{\Omega} a(x) f_{2}^{\prime}(q(x)+s)|u|^{r+1} d x\right)^{\frac{r}{r+1}} \\
& \leq(\ln b)^{-\frac{1}{r+1}} \int_{\Omega} a(x) \ln ^{\beta+1}(b+q(x)+s)\left|\partial_{t} u\right|^{r+1} d x-r(\ln b)^{-\frac{1}{r+1}} \int_{\Omega} a(x) f_{2}^{\prime}(q(x)+s)|u|^{r+1} d x
\end{aligned}
$$

Thus

$$
\begin{aligned}
& \int_{t}^{t+T} \int_{\Omega} a(x) f(q(x)+s)\left|\partial_{t} u\right|^{r-1} u \partial_{t} u d x d s+(r+1) \int_{t}^{t+T} \int_{\Omega} a(x) f_{2}(q(x)+s)|u|^{r-1} u \partial_{t} u d x d s \\
& \leq(r+1)(\ln b)^{-\frac{1}{r+1}} \int_{t}^{t+T} \int_{\Omega} a(x) \ln ^{\beta+1}(b+q(x)+s)\left|\partial_{t} u\right|^{r+1} d x d s \\
& -(r+1)(\ln b)^{-\frac{1}{r+1}} \int_{t}^{t+T} \int_{\Omega} a(x) f_{2}^{\prime}(q(x)+s)|u|^{r+1} d x d d s .
\end{aligned}
$$


Collecting the inequalities above, making some arrangement in (3.5) and integrating the result between $t$ and $t+T$, we end up with

$$
\begin{aligned}
& X(t+T)-X(t)+\left(\frac{1}{2}-(1+\beta) k\right) \int_{t}^{t+T} \int_{\Omega} f(q(x)+s)\left(|\nabla u|^{2}+\left|\partial_{t} u\right|^{2}\right) d x d s \\
& -\left(\frac{k_{1}}{4}-\frac{2(1+|\beta|)}{\epsilon_{0}}\right) \int_{t}^{t+T} \int_{\Omega} a(x) f_{1}^{\prime}(q(x)+s)|u(s)|^{2} d x d s \\
& -\left(1-(r+1)(\ln b)^{-\frac{1}{r+1}}\right) \int_{t}^{t+T} \int_{\Omega} a(x) f_{2}^{\prime}(q(x)+s)|u|^{r+1} d x d s \\
& +\left(k-(r+1)(\ln b)^{-\frac{1}{r+1}}\right) \int_{t}^{t+T} \int_{\Omega} a(x) \ln ^{\beta+1}(b+q(x)+s)\left|\partial_{t} u\right|^{r+1} d x d s \\
& \leq\left(3+\frac{1}{2}\left\|\nabla \chi^{2}\right\|_{\infty}\right)\left(\int_{t}^{t+T} \int_{\Omega \cap B_{2 L}} f(q(x)+s)\left(|u|^{2}+|\nabla u|^{2}+\left|\partial_{t} u\right|^{2}\right) d x d s\right) \\
& +\left(\frac{2}{\epsilon_{0}}+\frac{8(1+|\beta|)}{\epsilon_{0}^{2} k_{1}}+8 k_{1}\right) \int_{t}^{t+T} \int_{\Omega} a(x) f(q(x)+s)\left|\partial_{t} u\right|^{2} d x d s .
\end{aligned}
$$

Using the fact that $k=\frac{1}{4(\beta+1)}$ and

$$
\ln b \geq \max \left((2(r+1))^{r+1},(8(r+1)(\beta+1))^{r+1}\right)
$$

we obtain (3.4) .

3.2. Proof of Theorem 1. We assume that Hyp A holds and $\omega$ satisfies the GCC. We set $\gamma=\beta+1$. Let $u$ be a solution of (1.1) with initial data in $H_{0}^{1}(\Omega) \cap H^{2}(\Omega) \times H_{0}^{1}(\Omega)$ such that

$$
E_{\varphi}(u)(0)=\int_{\Omega} \ln ^{\beta+1}(1+q(x))\left(\left|\nabla u_{0}\right|^{2}+\left|u_{1}\right|^{2}\right) d x<\infty .
$$

Let $T>0$ such that the observability estimate (3.1) holds. First we estimate the first term of the RHS of (3.4) . Using the observability estimate (3.1), we see that

$$
\begin{aligned}
& X(t+T)-X(t)+\left(\frac{1}{4}-\left(3+\left\|\nabla \chi^{2}\right\|_{\infty}\right) \delta\right) \int_{t}^{t+T} \int_{\Omega} f(q(x)+s)\left(|\nabla u|^{2}+\left|\partial_{t} u\right|^{2}\right) d x d s \\
& -\left(\frac{k_{1}}{4}-\frac{2(1+|\beta|)}{\epsilon_{0}}-\left(3+\left\|\nabla \chi^{2}\right\|_{\infty}\right) C_{T, \delta}\right) \int_{t}^{t+T} \int_{\Omega} a(x) f_{1}^{\prime}(q(x)+s)|u|^{2} d x d s \\
& -\frac{1}{2} \int_{t}^{t+T} \int_{\Omega} a(x) f_{2}^{\prime}(q(x)+s)|u|^{r+1} d x d s \\
& +\frac{1}{8(\beta+1)} \int_{t}^{t+T} \int_{\Omega} a(x) \ln ^{\beta+1}(b+q(x)+s)\left|\partial_{t} u\right|^{r+1} d x d s \\
& \leq k_{3} \int_{t}^{t+T} \int_{\Omega} a(x) f(q(x)+s)\left(\left|\partial_{t} u\right|^{2}+\left|\partial_{t} u\right|^{2 r}\right) d x d s,
\end{aligned}
$$

for every $t \geq 0$, where $k_{3}=2\left(\frac{1}{\epsilon_{0}}+\frac{4(1+|\beta|)}{\epsilon_{0}^{2} k_{1}}+4 k_{1}+2\left(3+\left\|\nabla \chi^{2}\right\|_{\infty}\right) C_{T, \delta}\right)$. 
On the other hand, using Young's inequality we get

$$
\begin{aligned}
& X(t) \leq\left(\frac{k_{1}}{2}+\frac{1}{\epsilon_{0} \epsilon}\right) \int_{\Omega} a(x) f_{1}(q(x)+t)|u(t)|^{2} d x \\
& +(k+\epsilon) \int_{\Omega} \ln ^{\beta+1}(b+q(x)+t)\left(|\nabla u(t)|^{2}+\left|\partial_{t} u(t)\right|^{2}\right) d x \\
& +\int_{\Omega} a(x) f_{2}(q(x)+t)|u(t)|^{r+1} d x
\end{aligned}
$$

and

$$
\begin{aligned}
& X(t) \geq\left(\frac{k_{1}}{2}-\frac{1}{\epsilon_{0} \epsilon}\right) \int_{\Omega} a(x) f_{1}(q(x)+t)|u(t)|^{2} d x \\
& +(k-\epsilon) \int_{\Omega} \ln ^{\beta+1}(b+q(x)+t)\left(|\nabla u(t)|^{2}+\left|\partial_{t} u(t)\right|^{2}\right) d x \\
& +\int_{\Omega} a(x) f_{2}(q(x)+t)|u(t)|^{r+1} d x
\end{aligned}
$$

for all $\epsilon>0$. We choose (by taking into account of the order below)

$$
\begin{aligned}
& \delta \text { such that } \frac{1}{4}-\left(3+\left\|\nabla \chi^{2}\right\|_{\infty}\right) \delta=\frac{1}{8} \\
& \epsilon \text { such that } k-\epsilon \geq \frac{1}{16(\beta+1)}, \\
& k_{1} \text { such that } \frac{k_{1}}{2}-\frac{1}{\epsilon_{0} \epsilon} \geq 1 \text { and } \frac{k_{1}}{4}-\frac{2(1+|\beta|)}{\epsilon_{0}}-\left(3+\left\|\nabla \chi^{2}\right\|_{\infty}\right) C_{T, \delta} \geq 1 .
\end{aligned}
$$

Therefore

$$
\begin{aligned}
& X(t) \geq \int_{\Omega} a(x) f_{1}(q(x)+t)|u(t)|^{2} d x \\
& +\frac{1}{16(\beta+1)} \int_{\Omega} \ln ^{\beta+1}(b+q(x)+t)\left(|\nabla u(t)|^{2}+\left|\partial_{t} u(t)\right|^{2}\right) d x \\
& +\int_{\Omega} a(x) f_{2}(q(x)+t)|u(t)|^{r+1} d x .
\end{aligned}
$$

and

$$
\begin{aligned}
& X(t+T)-X(t)+\frac{1}{8} \int_{t}^{t+T} \int_{\Omega} f(q(x)+s)\left(|\nabla u|^{2}+\left|\partial_{t} u\right|^{2}\right) d x d s \\
& -\int_{t}^{t+T} \int_{\Omega} a(x) f_{1}^{\prime}(q(x)+s)|u|^{2} d x d s-\frac{1}{2} \int_{t}^{t+T} \int_{\Omega} a(x) f_{2}^{\prime}(q(x)+s)|u|^{r+1} d x d s \\
& +\frac{1}{8(\beta+1)} \int_{t}^{t+T} \int_{\Omega} a(x) \ln ^{\beta+1}(b+q(x)+s)\left|\partial_{t} u\right|^{r+1} d x d s \\
& \leq k_{3} \int_{t}^{t+T} \int_{\Omega} a(x) f(q(x)+s)\left(\left|\partial_{t} u\right|^{2}+\left|\partial_{t} u\right|^{2 r}\right) d x d s
\end{aligned}
$$


for every $t \geq 0$. Thus

$$
\begin{aligned}
& X(n T)+\frac{1}{8} \int_{0}^{n T} \int_{\Omega} f(q(x)+s)\left(|\nabla u|^{2}+\left|\partial_{t} u\right|^{2}\right) d x d s \\
& -\int_{0}^{n T} \int_{\Omega} a(x) f_{1}^{\prime}(q(x)+s)|u|^{2} d x d s-\frac{1}{2} \int_{0}^{n T} \int_{\Omega} a(x) f_{2}^{\prime}(q(x)+s)|u|^{r+1} d x d s \\
& +\frac{1}{8(\beta+1)} \int_{0}^{n T} \int_{\Omega} a(x) \ln ^{\beta+1}(b+q(x)+s)\left|\partial_{t} u\right|^{r+1} d x d s \\
& \leq k_{3} \int_{0}^{n T} \int_{\Omega} a(x) f(q(x)+s)\left(\left|\partial_{t} u\right|^{2}+\left|\partial_{t} u\right|^{2 r}\right) d x d s+X(0), \text { for all } n \in \mathbb{N} .
\end{aligned}
$$

Using proposition 1, we deduce that

$$
X(0) \leq C I_{0}
$$

where $I_{0}$ is defined in the statement of theorem 1 .

Combining (3.11) and (3.12), we obtain

$$
\begin{aligned}
& X(n T)+\frac{1}{8} \int_{0}^{n T} \int_{\Omega} f(q(x)+s)\left(|\nabla u|^{2}+\left|\partial_{t} u\right|^{2}\right) d x d s \\
& -\int_{0}^{n T} \int_{\Omega} a(x) f_{1}^{\prime}(q(x)+s)|u|^{2} d x d s-\frac{1}{2} \int_{0}^{n T} \int_{\Omega} a(x) f_{2}^{\prime}(q(x)+s)|u|^{r+1} d x d s \\
& +\frac{1}{8(\beta+1)} \int_{0}^{n T} \int_{\Omega} a(x) \ln ^{\beta+1}(b+q(x)+s)\left|\partial_{t} u\right|^{r+1} d x d s \\
& \leq k_{4}\left(\int_{0}^{n T} \int_{\Omega} a(x) f(q(x)+s)\left(\left|\partial_{t} u\right|^{2}+\left|\partial_{t} u\right|^{2 r}\right) d x d s+I_{0}\right), \text { for all } n \in \mathbb{N} .
\end{aligned}
$$

for some $k_{4}>0$. The next step is to control the first term of the RHS of the estimate above by the last term of the LHS. We remind that

$$
p= \begin{cases}2(r+1) & \text { if } d \leq 3 \\ \frac{2 d}{d-2} & \text { if } d \geq 4\end{cases}
$$


We have $r+1<2 r<p$, using interpolation inequality and Young's inequality, we obtain

$$
\begin{aligned}
& \int_{0}^{n T} \int_{\Omega} a(x) f(q(x)+s)\left|\partial_{t} u\right|^{2 r} d x d s \\
& \leq \int_{0}^{n T} f(s) \int_{\Omega} a(x)\left|\partial_{t} u\right|^{2 r} d x d s \\
& \leq \int_{0}^{n T} f(s)\left(\int_{\Omega} a(x)\left|\partial_{t} u\right|^{r+1} d x\right)^{\frac{p-2 r}{p-r-1}}\left(\int_{\Omega} a(x)\left|\partial_{t} u\right|^{p} d x\right)^{\frac{r-1}{p-r-1}} d s \\
& \leq\left(\|a\|_{L^{\infty}}\left\|\partial_{t} u\right\|_{L^{\infty}\left(\mathbb{R}_{+}, L^{p}(\Omega)\right)}^{p} \int_{0}^{n T}(f(s))^{\frac{p-r-1}{r-1}}(\ln (b+s))^{-\frac{(\beta+1)(p-2 r)}{r-1}} d s\right)^{\frac{r-1}{p-r-1}} \\
& \times\left(\int_{0}^{n T} \ln ^{\beta+1}(b+s) \int_{\Omega} a(x)\left|\partial_{t} u\right|^{r+1} d x d s\right)^{\frac{p-2 r}{p-r-1}} \\
& \leq \frac{\epsilon^{-\frac{p-2 r}{r-1}}(r-1)\|a\|_{L^{\infty}}\left\|\partial_{t} u\right\|_{L^{\infty}\left(\mathbb{R}_{+}, L^{p}(\Omega)\right)}^{p}}{p-r-1} \int_{0}^{+\infty}(b+s)^{-\frac{p-r-1}{r-1}}(\ln (b+s))^{\beta-\frac{p-2 r}{r-1}} d s \\
& +\frac{\epsilon(p-2 r)}{p-r-1} \int_{0}^{n T} \int_{\Omega} a(x) \ln ^{\beta+1}(b+q(x)+s)\left|\partial_{t} u\right|^{r+1} d x d s,
\end{aligned}
$$

for all $\epsilon>0$. Thus using (1.3) and Sobolev imbedding $H^{1} \hookrightarrow L^{p}$, we get

$$
\begin{aligned}
& \int_{0}^{n T} \int_{\Omega} a(x) f(q(x)+s)\left|\partial_{t} u\right|^{2 r} d x d s \\
& \leq \epsilon^{-\frac{p-2 r}{r-1}} C\|a\|_{L^{\infty}}\left(\left\|u_{0}\right\|_{H^{2}}^{2}+\left\|u_{1}\right\|_{H^{1}}^{2}+\left\|u_{1}\right\|_{H^{1}}^{2 r}\right)^{\frac{p}{2}} \\
& +\frac{\epsilon(p-2 r)}{p-r-1} \int_{0}^{n T} \int_{\Omega} a(x)(\ln (b+q(x)+s))^{\beta+1}\left|\partial_{t} u\right|^{r+1} d x d s
\end{aligned}
$$

for all $\epsilon>0$. To estimate the last term, first we use Holder's inequality

$$
\begin{aligned}
& \int_{0}^{n T} \int_{\Omega} a(x) f(q(x)+s)\left|\partial_{t} u\right|^{2} d x d s \\
& \leq\left(\|a\|_{L^{\infty}} \int_{0}^{n T} \int_{\Omega}(f(q(x)+s))^{\frac{r+1}{r-1}} \ln ^{-\frac{2(\beta+1)}{r-1}}(b+q(x)+s) d x d s\right)^{\frac{r-1}{r+1}} \\
& \times\left(\int_{0}^{n T} \int_{\Omega} a(x) \ln ^{\beta+1}(b+q(x)+s)\left|\partial_{t} u\right|^{r+1} d x d s\right)^{\frac{2}{r+1}} \\
& \leq\left(\|a\|_{L^{\infty}} \int_{0}^{+\infty} \int_{\Omega}(b+q(x)+s)^{-\frac{r+1}{r-1}} \ln ^{\beta-\frac{2}{r-1}}(b+q(x)+s) d x d s\right)^{\frac{r-1}{r+1}} \\
& \times\left(\int_{0}^{n T} \int_{\Omega} a(x) \ln ^{\beta+1}(b+q(x)+s)\left|\partial_{t} u\right|^{r+1} d x d s\right)^{\frac{2}{r+1}} .
\end{aligned}
$$


By Young's inequality, we end up with

$$
\begin{aligned}
& \int_{0}^{n T} \int_{\Omega} a(x) f(q(x)+s)\left|\partial_{t} u\right|^{2} d x d s \\
& \leq \frac{(r-1) \epsilon^{-\frac{2}{r-1}}\|a\|_{L^{\infty}}}{r+1} \int_{0}^{+\infty} \int_{\Omega}(b+q(x)+s)^{-\frac{r+1}{r-1}} \ln ^{\beta-\frac{2}{r-1}}(b+q(x)+s) d x d s \\
& +\frac{2 \epsilon}{r+1} \int_{0}^{n T} \int_{\Omega} a(x) \ln ^{\beta+1}(b+q(x)+s)\left|\partial_{t} u\right|^{r+1} d x d s \\
& \leq C\|a\|_{L^{\infty}} \frac{(r-1) \epsilon^{-\frac{2}{r-1}}}{r+1} \int_{0}^{+\infty} \int_{0}^{+\infty} \ln ^{\beta-\frac{2}{r-1}}(b+y+s)(b+y+s)^{-\frac{r+1}{r-1}+d-1} d y d s \\
& +\frac{2 \epsilon}{r+1} \int_{0}^{n T} \int_{\Omega} a(x) \ln ^{\beta+1}(b+q(x)+s)\left|\partial_{t} u\right|^{r+1} d x d s,
\end{aligned}
$$

for all $\epsilon>0$. In view of the fact that

$$
\begin{array}{ll}
-\frac{r+1}{r-1}+d<-1 & \text { if } 1<r<1+\frac{2}{d} \\
\beta-\frac{2}{r-1}<-1 \text { and }-\frac{r+1}{r-1}+d=-1 & \text { if } r=1+\frac{2}{d},
\end{array}
$$

we see that

$$
\begin{aligned}
& \int_{0}^{n T} \int_{\Omega} a(x) f(q(x)+s)\left|\partial_{t} u\right|^{2} d x d s \\
& \leq C \epsilon^{-\frac{2}{r-1}}\|a\|_{L^{\infty}}+\frac{2 \epsilon}{r+1} \int_{0}^{n T} \int_{\Omega} a(x) \ln ^{\beta+1}(b+q(x)+s)\left|\partial_{t} u\right|^{r+1} d x d s,
\end{aligned}
$$

for all $\epsilon>0$. We choose $\epsilon$ such that

$$
\frac{1}{8(\beta+1)}-k_{4} \epsilon\left(\frac{p-2 r}{p-r-1}+\frac{2}{r+1}\right) \geq \frac{1}{16(\beta+1)} .
$$

We conclude that there exists a positive constant $C_{1}$ such that

$$
\begin{aligned}
& X(n T)+\frac{1}{8} \int_{0}^{n T} \int_{\Omega} f(q(x)+s)\left(|\nabla u|^{2}+\left|\partial_{t} u\right|^{2}\right) d x d s \\
& -\int_{0}^{n T} \int_{\Omega} a(x) f_{1}^{\prime}(q(x)+s)|u|^{2} d x d s-\frac{1}{2} \int_{0}^{n T} \int_{\Omega} a(x) f_{2}^{\prime}(q(x)+s)|u|^{r+1} d x d s \\
& +\frac{1}{16(\beta+1)} \int_{0}^{n T} \int_{\Omega} a(x) \ln ^{\beta+1}(b+q(x)+s)\left|\partial_{t} u\right|^{r+1} d x d s \leq C_{1} I_{0}, \text { for all } n \in \mathbb{N} .
\end{aligned}
$$

Therefore we obtain

$$
\begin{aligned}
& \frac{1}{8} \int_{0}^{\infty} \int_{\Omega} f(q(x)+s)\left(|\nabla u|^{2}+\left|\partial_{t} u\right|^{2}\right) d x d s \\
& -\int_{0}^{+\infty} \int_{\Omega} a(x) f_{1}^{\prime}(q(x)+s)|u|^{2} d x d s-\frac{1}{2} \int_{0}^{+\infty} \int_{\Omega} a(x) f_{2}^{\prime}(q(x)+s)|u|^{r+1} d x d s \\
& +\frac{1}{16(\beta+1)} \int_{0}^{+\infty} \int_{\Omega} a(x) \ln ^{\beta+1}(b+q(x)+s)\left|\partial_{t} u\right|^{r+1} d x d s \leq C_{1} I_{0} .
\end{aligned}
$$


Now using the weighted energy estimate (2.3), we infer that

$$
\begin{aligned}
& E_{\varphi}(u)(t)=\int_{\Omega} \varphi(q(x)+s)\left(|\nabla u(s)|^{2}+\left|\partial_{t} u(s)\right|^{2}\right) \\
& \leq E_{\varphi}(u)(0)+(\beta+1) \int_{0}^{\infty} \int_{\Omega} f(q(x)+s)\left(|\nabla u(s)|^{2}+\left|\partial_{t} u(s)\right|^{2}\right) d x d s \\
& \leq C_{0} I_{0},
\end{aligned}
$$

for some positive constant $C_{0}$. The sought estimate follows from the estimate above and the fact that

$$
\ln ^{\beta+1}(2+t) E_{u}(t) \leq E_{\varphi}(u)(t) .
$$

\section{Proof of Theorem 2}

4.1. Preliminary results. Throughout this section we use the following notations: Let $\beta$ be a real number such that $0<1+\beta<\tau$, where

$$
\tau=\frac{r \delta_{0}^{\frac{r^{2}+1}{r}}(\lambda+1)^{r-1}(r+1)^{r}}{\delta_{0}^{\frac{r+1}{r}}+\delta_{0}^{r}(\lambda+1)^{r-1}(r+1)^{r}\left(r \delta_{0}(\lambda+1)(r+1)+\delta_{0}^{\frac{1}{r}}\right)},
$$

$\lambda$ any positive constant and

$$
\delta_{0}=(\lambda+1)^{\frac{r^{2}}{r^{2}-1}}(r+1)^{-\frac{r}{r-1}} .
$$

We take $\varphi(s)=(1+\alpha s)^{\beta+1}$ where

$$
\alpha=\frac{r k^{r}(r+1)+\delta_{0}^{\frac{1}{r}}}{k^{r} \delta_{0}^{\frac{1}{r}}(r+1)(r-\tau)},
$$

and

$$
k=(1+\lambda)(r+1) \delta_{0} .
$$

Finally, let $\psi \in C_{c}^{\infty}\left(\mathbb{R}^{d}\right)$ such that $0 \leq \psi \leq 1$ and

$$
\psi(x)=\left\{\begin{array}{ll}
1 & \text { for }|x| \leq L \\
0 & \text { for }|x| \geq 2 L
\end{array} .\right.
$$

Proposition 4. We assume that Hyp A holds and $(\omega, T)$ geometrically controls $\Omega$. Let $\delta>0$, $R_{0}>L$ and $-1<\beta \leq 0$. There exists $C_{T, \delta}=C\left(T, \delta, R_{0}, \alpha, \beta\right)>0$, such that the following inequality

$$
\begin{aligned}
& \int_{t}^{t+T} \int_{\Omega \cap B_{R_{0}}}(1+\alpha(q(x)+s))^{\beta}\left(|u|^{2}+|\nabla u|^{2}+\left|\partial_{t} u\right|^{2}\right) d x d s \\
& \leq C_{T, \delta} \int_{t}^{t+T} \int_{\Omega} a(x)(1+\alpha(q(x)+s))^{\beta}\left(\left|\partial_{t} u\right|^{2}+\left|\partial_{t} u\right|^{2 r}\right) d x d s \\
& +C_{T, \delta} \int_{t}^{t+T} \int_{\Omega} a(x)(1+\alpha(q(x)+s))^{\beta-2}|u|^{2} d x d s \\
& +\delta \int_{t}^{t+T} \int_{\Omega}(1+\alpha(q(x)+s))^{\beta}\left(|\nabla u|^{2}+\left|\partial_{t} u\right|^{2}\right) d x d s,
\end{aligned}
$$

holds for every $t \geq 0$ and for all $u$ solution of (1.1) with initial data $\left(u_{0}, u_{1}\right)$ in $H_{0}^{1}(\Omega) \cap$ $H^{2}(\Omega) \times H_{0}^{1}(\Omega)$. 
Proof. We set

$$
f(s)=(1+\alpha s)^{\beta}
$$

In view of $f \in L^{\infty}\left(\mathbb{R}_{+}\right)$, we have $E_{f}(u)(0)<\infty$. On the other hand, it is clear that $f^{\prime} \in L^{\infty}\left(\mathbb{R}_{+}\right)$and there exists a positive constant $K$, such that

$$
\sup _{\mathbb{R}_{+}}\left|\frac{f^{\prime \prime}(t)}{f^{\prime}(t)}\right| \leq K
$$

In addition the function $t \longmapsto\left|\frac{f^{\prime}(t)}{f(t)}\right|$ is decreasing and $\lim _{t \rightarrow+\infty}\left|\frac{f^{\prime}(t)}{f(t)}\right|=0$. Moreover there exists $C>0$, such that

$$
\frac{\left(f^{\prime}(t)\right)^{2}}{f(t)} \leq C\left(-f^{\prime}(t)\right), \text { for all } t \geq 0
$$

Since

$$
\partial_{t} u \in L^{\infty}\left(\mathbb{R}_{+}, H_{0}^{1}(\Omega)\right),
$$

then from Sobolev imbedding, we deduce that

$$
\sqrt{a(x)(1+\alpha(q(x)+s))^{\beta}}\left|\partial_{t} u\right|^{r} \in L_{l o c}^{2}\left(\mathbb{R}_{+}, L^{2}(\Omega)\right) .
$$

By taking into account of the results above, we can use proposition 2 and we obtain (4.1). This finishes the proof of the proposition.

In order to prove theorem 2 we need the following result.

Lemma 3. Let $u$ be a solution of (1.1) with initial data in $H_{0}^{1}(\Omega) \cap H^{2}(\Omega) \times H_{0}^{1}(\Omega)$ such that

$$
E_{\varphi}(u)(0)=\left\|(1+\alpha q)^{\frac{1+\beta}{2}} \nabla u_{0}\right\|_{L^{2}}^{2}+\left\|(1+\alpha q)^{\frac{1+\beta}{2}} u_{1}\right\|_{L^{2}}^{2}<+\infty .
$$

We set $\chi=1-\psi$ and

$$
\begin{aligned}
& X(t)=\int_{\Omega}(1+\alpha(q(x)+t))^{\beta} \chi^{2}(x) u(t) \partial_{t} u(t) d x+\frac{k_{1}}{2} \int_{\Omega}(1+\alpha(q(x)+t))^{\beta-1} a(x)|u(t)|^{2} d x \\
& +\int_{\Omega} a(x)(1+\alpha(q(x)+t))^{\beta-r+1}|u(t)|^{r+1} d x+\frac{k}{2} \int_{\Omega}(1+\alpha(q(x)+t))^{\beta+1}\left(|\nabla u|^{2}+\left|\partial_{t} u\right|^{2}\right) d x,
\end{aligned}
$$

where $k_{1}>0$. Then

$$
\begin{aligned}
& X(t+T)-X(t)+\frac{1-k \alpha(1+\beta)}{2} \int_{t}^{t+T} \int_{\Omega}(1+\alpha(q(x)+s))^{\beta}\left(|\nabla u|^{2}+\left|\partial_{t} u\right|^{2}\right) d x d s \\
& +\left(\frac{k_{1} \alpha(1-\beta)}{4}-\frac{\beta^{2} \alpha^{2}}{\epsilon_{0} \epsilon}\right) \int_{t}^{t+T} \int_{\Omega} a(x)(1+\alpha(q(x)+s))^{\beta-2}|u(t)|^{2} d x d s \\
& +\lambda \delta_{0} \int_{t}^{t+T} \int_{\Omega} a(x)(1+\alpha(q(x)+s))^{\beta+1}\left|\partial_{t} u\right|^{r+1} d x d s \\
& \leq\left(3+\left\|\nabla \chi^{2}\right\|_{\infty}\right) \int_{t}^{t+T} \int_{\Omega \cap B_{2 L}}(1+\alpha(q(x)+s))^{\beta}\left(|u|^{2}+|\nabla u|^{2}+\left|\partial_{t} u\right|^{2}\right) d x d s \\
& +\left(\frac{2}{\epsilon_{0}}+\frac{8 k_{1}}{\alpha(1-\beta)}+\frac{8 \beta^{2} \alpha}{\epsilon_{0}^{2} k_{1}(1-\beta)}\right) \int_{t}^{t+T} \int_{\Omega} a(x)(1+\alpha(q(x)+s))^{\beta}\left|\partial_{t} u\right|^{2} d x d s,
\end{aligned}
$$

for all $t \geq 0$, where $\lambda$ any positive constant. 
Proof. We have

$$
\int_{\Omega} \varphi(q(x))\left(\left|\nabla u_{0}\right|^{2}+\left|u_{1}\right|^{2}\right) d x<\infty
$$

Then from (2.3), we infer

$$
\begin{aligned}
& E_{\varphi}(u)(t+T)+\int_{t}^{t+T} \int_{\Omega} a(x)(1+\alpha(q(x)+s))^{\beta+1}\left|\partial_{t} u\right|^{r+1} d x d s \\
& \leq E_{\varphi}(u)(t)+(\beta+1) \alpha \int_{t}^{t+T} \int_{\Omega}(1+\alpha(q(x)+s))^{\beta}\left(|\nabla u|^{2}+\left|\partial_{t} u\right|^{2}\right) d x d s .
\end{aligned}
$$

We set

$$
\begin{aligned}
& X_{0}(t)=\int_{\Omega}(1+\alpha(q(x)+t))^{\beta} \chi^{2}(x) u(t) \partial_{t} u(t) d x+\frac{k_{1}}{2} \int_{\Omega}(1+\alpha(q(x)+t))^{\beta-1} a(x)|u(t)|^{2} d x \\
& +\int_{\Omega} a(x)(1+\alpha(q(x)+t))^{\beta-r+1}|u(t)|^{r+1} d x .
\end{aligned}
$$

Using the fact that $u$ is a solution of (1.1), we deduce that

$$
\begin{aligned}
& \frac{d}{d t} X_{0}(t)=\int_{\Omega}\left(\left|\partial_{t} u(t)\right|^{2}-|\nabla u(t)|^{2}-a(x)\left|\partial_{t} u(t)\right|^{r-1} u(t) \partial_{t} u(t)\right) \chi^{2}(x)(1+\alpha(q(x)+t))^{\beta} d x \\
& -\int_{\Omega}(1+\alpha(q(x)+t))^{\beta} u(t) \nabla \chi^{2}(x) \nabla u(t)+\beta \alpha(1+\alpha(q(x)+t))^{\beta-1} \chi^{2}(x) u(t) \frac{x \cdot \nabla u(t)}{q(x)} d x \\
& +\beta \alpha \int_{\Omega}(1+\alpha(q(x)+t))^{\beta-1} \chi^{2}(x) u(t) \partial_{t} u(t) d x \\
& +k_{1}\left(\int_{\Omega} a(x)(1+\alpha(q(x)+t))^{\beta-1} u(t) \partial_{t} u(t) d x+\frac{\beta-1}{2} \alpha \int_{\Omega} a(x)(1+\alpha(q(x)+t))^{\beta-2}|u|^{2} d x\right) \\
& +(\beta+1-r) \alpha \int_{\Omega} a(x)(1+\alpha(q(x)+t))^{\beta-r}|u|^{r+1} d x \\
& +(r+1) \int_{\Omega} a(x)(1+\alpha(q(x)+t))^{\beta-r+1}|u|^{r-1} u \partial_{t} u d x .
\end{aligned}
$$

We note that $\|\chi\|_{\infty} \leq 1$. Using Young's inequality and the fact that the support of $\chi$ is contained in $\{|x| \geq L\}$ and

$$
a(x)>\epsilon_{0}>0 \text { for }|x| \geq L
$$

we infer that

$$
\begin{aligned}
& \left|\alpha \beta \int_{\Omega}(1+\alpha q(x)+\alpha t)^{\beta-1} \chi^{2}(x) u(t) \partial_{t} u(t) d x\right| \\
& \leq \frac{k_{1} \alpha(1-\beta)}{8} \int_{\Omega} a(x)(1+\alpha(q(x)+t))^{\beta-2}|u(t)|^{2} d x+\frac{8 \beta^{2} \alpha}{\epsilon_{0}^{2} k_{1}(1-\beta)} \int_{\Omega} a(x)(1+\alpha(q(x)+t))^{\beta}\left|\partial_{t} u(t)\right|^{2} d x
\end{aligned}
$$

and

$$
\begin{aligned}
& k_{1}\left|\int_{\Omega} a(x)(1+\alpha(q(x)+t))^{\beta-1} u(t) \partial_{t} u(t) d x\right| \\
& \leq \frac{k_{1} \alpha(1-\beta)}{8} \int_{\Omega} a(x)(1+\alpha(q(x)+t))^{\beta-2}|u(t)|^{2} d x+\frac{8 k_{1}}{\alpha(1-\beta)} \int_{\Omega} a(x)(1+\alpha(q(x)+t))^{\beta}\left|\partial_{t} u(t)\right|^{2} d x .
\end{aligned}
$$


Using the same arguments, we also deduce that

$$
\begin{aligned}
& \left|\int_{\Omega} \beta \alpha(1+\alpha(q(x)+t))^{\beta-1} \chi^{2}(x) u(t) \frac{x \cdot \nabla u(t)}{q(x)} d x\right| \\
& \leq \frac{\beta^{2} \alpha^{2}}{\epsilon_{0} \epsilon} \int_{\Omega} a(x)(1+\alpha(q(x)+t))^{\beta-2}|u(t)|^{2} d x+\epsilon \int_{\Omega}(1+\alpha(q(x)+t))^{\beta}|\nabla u(t)|^{2} d x,
\end{aligned}
$$

for all $\epsilon>0$. Using the fact that the support of $\psi$ is contained in $\{|x| \leq 2 L\}$ and

$$
a(x)>\epsilon_{0}>0 \text { for }|x| \geq L
$$

we get

$$
\begin{aligned}
& \int_{\Omega} \chi^{2}(x)\left(\left|\partial_{t} u(t)\right|^{2}-|\nabla u(t)|^{2}\right)(1+\alpha(q(x)+t))^{\beta} d x \\
& =\int_{\Omega}\left(1-2 \psi(x)+\psi^{2}(x)\right)(1+\alpha(q(x)+t))^{\beta}\left(\left|\partial_{t} u(t)\right|^{2}-|\nabla u(t)|^{2}\right) d x \\
& \leq \frac{2}{\epsilon_{0}} \int_{\Omega} a(x)(1+\alpha(q(x)+t))^{\beta}\left|\partial_{t} u(t)\right|^{2} d x \\
& -\int_{\Omega}(1+\alpha(q(x)+t))^{\beta}\left(\left|\partial_{t} u(t)\right|^{2}+|\nabla u(t)|^{2}\right) d x \\
& +3 \int_{\Omega \cap B_{2 L}}(1+\alpha(q(x)+t))^{\beta}\left(\left|\partial_{t} u(t)\right|^{2}+|\nabla u(t)|^{2}\right) d x .
\end{aligned}
$$

We note that the support of $\nabla \chi^{2}$ is contained in $\{|x| \leq 2 L\}$, using Young's inequality, we deduce that

$$
\begin{aligned}
& \left|-\int_{\Omega}(1+\alpha(q(x)+t))^{\beta} u(t) \nabla \chi^{2}(x) \nabla u(t) d x\right| \\
& \leq \frac{1}{2}\left\|\nabla \chi^{2}\right\|_{\infty} \int_{\Omega \cap B_{2 L}}(1+\alpha(q(x)+t))^{\beta}\left(|u(t)|^{2}+|\nabla u(t)|^{2}\right) d x .
\end{aligned}
$$

Young's inequality combined with the fact that $\|\chi\|_{\infty} \leq 1$, gives

$$
\begin{aligned}
& \left.\left|\int_{\Omega} a(x)(1+\alpha(q(x)+t))^{\beta} \chi^{2}(x)\right| \partial_{t} u(t)\right|^{r-1} u \partial_{t} u(t) d x \mid \\
& \leq \frac{r k}{r+1} \int_{\Omega} a(x)(1+\alpha(q(x)+t))^{\beta+1}\left|\partial_{t} u(t)\right|^{r+1} d x+\frac{k^{-r}}{r+1} \int_{\Omega} a(x)(1+\alpha(q(x)+t))^{\beta-r}|u(t)|^{r+1} d x
\end{aligned}
$$

and

$$
\begin{aligned}
& \left.(r+1)\left|\int_{\Omega} a(x)(1+\alpha(q(x)+t))^{\beta-r+1}\right| u(t)\right|^{r-1} u \partial_{t} u(t) d x \mid \\
& \leq \delta_{0} \int_{\Omega} a(x)(1+\alpha(q(x)+t))^{\beta+1}\left|\partial_{t} u(t)\right|^{r+1} d x+r \delta_{0}^{-\frac{1}{r}} \int_{\Omega} a(x)(1+\alpha(q(x)+t))^{\beta-r}|u(t)|^{r+1} d x .
\end{aligned}
$$


By taking into account of the estimates above, making some arrangement in (4.4) and integrating the result between $t$ and $t+T$, we obtain

$$
\begin{aligned}
& X(t+T)-X(t)+(1-\epsilon-(1+\beta) k \alpha) \int_{t}^{t+T} \int_{\Omega}(1+\alpha(q(x)+s))^{\beta}\left(|\nabla u|^{2}+\left|\partial_{t} u\right|^{2}\right) d x d s \\
& +\left(\frac{k_{1} \alpha(1-\beta)}{4}-\frac{\beta^{2} \alpha^{2}}{\epsilon_{0} \epsilon}\right) \int_{t}^{t+T} \int_{\Omega} a(x)(1+\alpha(q(x)+s))^{\beta-2}|u|^{2} d x d s \\
& +\left(\left(\alpha-\delta_{0}^{-\frac{1}{r}}\right) r-(\beta+1) \alpha-\frac{k^{-r}}{r+1}\right) \int_{t}^{t+T} \int_{\Omega} a(x)(1+\alpha(q(x)+s))^{\beta-r}|u|^{r+1} d x d s \\
& +\left(k-\frac{k r}{r+1}-\delta_{0}\right) \int_{t}^{t+T} \int_{\Omega} a(x)(1+\alpha(q(x)+s))^{\beta+1}\left|\partial_{t} u\right|^{r+1} d x d s \\
& \leq\left(3+\left\|\nabla \chi^{2}\right\|_{\infty}\right) \int_{t}^{t+T} \int_{\Omega \cap B_{2 L}}(1+\alpha(q(x)+s))^{\beta}\left(|u|^{2}+|\nabla u|^{2}+\left|\partial_{t} u\right|^{2}\right) d x d s \\
& +\left(\frac{2}{\epsilon_{0}}+\frac{8 k_{1}}{\alpha(1-\beta)}+\frac{8 \beta^{2} \alpha}{\epsilon_{0}^{2} k_{1}(1-\beta)}\right) \int_{t}^{t+T} \int_{\Omega} a(x)(1+\alpha(q(x)+s))^{\beta}\left|\partial_{t} u\right|^{2} d x d s,
\end{aligned}
$$

for all $\epsilon>0$.

We have

$$
1-(\beta+1) k \alpha>1-\tau k \alpha=0 .
$$

So we can choose $\epsilon=\frac{1-\gamma k \alpha}{2}$. It is easy to see that

$$
\begin{aligned}
& \left(\alpha-\delta_{0}^{-\frac{1}{r}}\right) r-(\beta+1) \alpha-\frac{k^{-r}}{r+1} \\
& >\left(\alpha-\delta_{0}^{-\frac{1}{r}}\right) r-\tau \alpha-\frac{k^{-r}}{r+1}=0
\end{aligned}
$$

and

$$
k-\frac{k r}{r+1}-\delta_{0}=\lambda \delta_{0}
$$

Collecting the estimates above, we get (4.3) .

4.2. Proof of Theorem 2. We assume that Hyp A holds and $\omega$ satisfies the GCC. Let $u$ be a solution of (1.1) with initial data in $H_{0}^{1}(\Omega) \cap H^{2}(\Omega) \times H_{0}^{1}(\Omega)$ such that

$$
\left\|(1+\alpha q)^{\frac{\gamma}{2}} \nabla u_{0}\right\|_{L^{2}}^{2}+\left\|(1+\alpha q)^{\frac{\gamma}{2}} u_{1}\right\|_{L^{2}}^{2}<+\infty .
$$

We set $\gamma=1+\beta$. Using (4.3) and (4.1) and arguing as in the proof of theorem 1 we obtain

$$
\begin{aligned}
& X(t+T)-X(t)+\left(\frac{1-k \alpha(1+\beta)}{2}-\left(3+\left\|\nabla \chi^{2}\right\|_{\infty}\right) \delta\right) \int_{t}^{t+T} \int_{\Omega}(1+\alpha(q(x)+s))^{\beta}\left(|\nabla u|^{2}+\left|\partial_{t} u\right|^{2}\right) d x d s \\
& +\left(\frac{k_{1} \alpha(1-\beta)}{4}-\frac{\beta^{2} \alpha^{2}}{\epsilon_{0} \epsilon}-\left(3+\left\|\nabla \chi^{2}\right\|_{\infty}\right) C_{T, \delta}\right) \int_{t}^{t+T} \int_{\Omega} a(x)(1+\alpha(q(x)+s))^{\beta-2}|u|^{2} d x d s \\
& +\lambda \delta_{0} \int_{t}^{t+T} \int_{\Omega} a(x)(1+\alpha(q(x)+s))^{\beta+1}\left|\partial_{t} u\right|^{r+1} d x d s \\
& \leq k_{2} \int_{t}^{t+T} \int_{\Omega} a(x)(1+\alpha(q(x)+s))^{\beta}\left(\left|\partial_{t} u\right|^{2 r}+\left|\partial_{t} u\right|^{2}\right) d x d s,
\end{aligned}
$$

for all $t \geq 0$, and for some $k_{2}>0$. 
Using Young's inequality we get

$$
\begin{aligned}
& X(t) \leq\left(\frac{k_{1}}{2}+\frac{1}{2 \epsilon_{0} \epsilon}\right) \int_{\Omega} a(x)(1+\alpha(q(x)+t))^{\beta-1}|u(t)|^{2} d x \\
& +(k+\epsilon) \int_{\Omega}(1+\alpha(q(x)+t))^{\beta+1}\left(|\nabla u(t)|^{2}+\left|\partial_{t} u(t)\right|^{2}\right) d x \\
& +\int_{\Omega} a(x)(1+\alpha(q(x)+t))^{\beta-r+1}|u(t)|^{r+1} d x
\end{aligned}
$$

and

$$
\begin{aligned}
& X(t) \geq\left(\frac{k_{1}}{2}-\frac{1}{\epsilon_{0} \epsilon}\right) \int_{\Omega} a(x)(1+\alpha(q(x)+t))^{\beta-1}|u(t)|^{2} d x \\
& +(k-\epsilon) \int_{\Omega}(1+\alpha(q(x)+t))^{\beta+1}\left(|\nabla u(t)|^{2}+\left|\partial_{t} u(t)\right|^{2}\right) d x \\
& +\int_{\Omega} a(x)(1+\alpha(q(x)+t))^{\beta-r+1}|u(t)|^{r+1} d x
\end{aligned}
$$

for all $\epsilon>0$. We choose (by taking into account of the order below)

$\epsilon$ such that $k-\epsilon \geq \delta_{0}$

$\delta$ such that $\frac{1-k \alpha(1+\beta)}{2}-\left(3+\left\|\nabla \chi^{2}\right\|_{\infty}\right) \delta \geq \frac{1-k \alpha(1+\beta)}{4}$

$k_{1}$ such that $\frac{k_{1}}{2}-\frac{1}{2 \epsilon_{0} \epsilon} \geq \delta_{0}$ and $\frac{k_{1}(1-\beta)}{4}-\frac{2 \beta^{2}}{\epsilon_{0} \delta_{0}}-\left(3+\left\|\nabla \chi^{2}\right\|_{\infty}\right) C_{T, \delta} \geq \delta_{0}$.

Therefore

$$
\begin{aligned}
& X(t) \geq \delta_{0} \int_{\Omega} a(x)(1+\alpha(q(x)+t))^{\beta-1}|u(t)|^{2} d x \\
& +\delta_{0} \int_{\Omega}(1+\alpha(q(x)+t))^{\beta+1}\left(|\nabla u(t)|^{2}+\left|\partial_{t} u(t)\right|^{2}\right) d x \\
& +\int_{\Omega} a(x)(1+\alpha(q(x)+t))^{\beta-r+1}|u(t)|^{r+1} d x
\end{aligned}
$$

and

$$
\begin{aligned}
& X(t+T)-X(t)+\frac{1-k \alpha(1+\beta)}{4} \int_{t}^{t+T} \int_{\Omega}(1+\alpha(q(x)+s))^{\beta}\left(|\nabla u|^{2}+\left|\partial_{t} u\right|^{2}\right) d x d s \\
& +\delta_{0} \int_{t}^{t+T} \int_{\Omega} a(x)(1+\alpha(q(x)+s))^{\beta-2}|u|^{2} d x d s \\
& +\lambda \delta_{0} \int_{t}^{t+T} \int_{\Omega} a(x)(1+\alpha(q(x)+s))^{\beta+1}\left|\partial_{t} u\right|^{r+1} d x d s \\
& \leq k_{2} \int_{t}^{t+T} \int_{\Omega} a(x)(1+\alpha(q(x)+s))^{\beta}\left(\left|\partial_{t} u\right|^{2 r}+\left|\partial_{t} u\right|^{2}\right) d x d s,
\end{aligned}
$$


for all $t \geq 0$. Thus

$$
\begin{aligned}
& X(n T)+\frac{1-k \alpha(1+\beta)}{4} \int_{0}^{n T} \int_{\Omega}(1+\alpha(q(x)+s))^{\beta}\left(|\nabla u|^{2}+\left|\partial_{t} u\right|^{2}\right) d x d s \\
& +\delta_{0} \int_{0}^{n T} \int_{\Omega} a(x)(1+\alpha(q(x)+s))^{\beta-2}|u|^{2} d x d s \\
& +\lambda \delta_{0} \int_{0}^{n T} \int_{\Omega} a(x)(1+\alpha(q(x)+s))^{\beta+1}\left|\partial_{t} u\right|^{r+1} d x d s \\
& \leq k_{2} \int_{0}^{n T} \int_{\Omega} a(x)(1+\alpha(q(x)+s))^{\beta}\left(\left|\partial_{t} u\right|^{2 r}+\left|\partial_{t} u\right|^{2}\right) d x d s+X(0), \text { for all } n \geq 1
\end{aligned}
$$

In view of proposition 1

$$
X(0) \leq C I_{1}
$$

where $I_{1}$ is defined in the statement of theorem 2 . Therefore there exists a positive constant $k_{3}$, such that

$$
\begin{aligned}
& X(n T)+\frac{1-k \alpha(1+\beta)}{4} \int_{0}^{n T} \int_{\Omega}(1+\alpha(q(x)+s))^{\beta}\left(|\nabla u|^{2}+\left|\partial_{t} u\right|^{2}\right) d x d s \\
& +\delta_{0} \int_{0}^{n T} \int_{\Omega} a(x)(1+\alpha(q(x)+s))^{\beta-2}|u|^{2} d x d s \\
& +\lambda \delta_{0} \int_{0}^{n T} \int_{\Omega} a(x)(1+\alpha(q(x)+s))^{\beta+1}\left|\partial_{t} u\right|^{r+1} d x d s \\
& \leq k_{3}\left(\int_{0}^{n T} \int_{\Omega} a(x)(1+\alpha(q(x)+s))^{\beta}\left(\left|\partial_{t} u\right|^{2}+\left|\partial_{t} u\right|^{2 r}\right) d x d s+I_{1}\right), \text { for all } n \in \mathbb{N} .
\end{aligned}
$$

As in the proof of theorem 1, we absorb the first term of the RHS of the estimate above by the last term of the LHS. Proceeding as the proof of theorem 1, we obtain

$$
\begin{aligned}
& \int_{0}^{n T} \int_{\Omega} a(x)(1+\alpha(q(x)+s))^{\beta}\left|\partial_{t} u\right|^{2 r} d x d s \leq C \epsilon^{-\frac{p-2 r}{r-1}}\|a\|_{L^{\infty}}\left(\left\|u_{0}\right\|_{H^{2}}^{2}+\left\|u_{1}\right\|_{H^{1}}^{2}+\left\|u_{1}\right\|_{H^{1}}^{2 r}\right)^{\frac{p}{2}} \\
& +\frac{\epsilon(p-2 r)}{p-r-1} \int_{0}^{n T} \int_{\Omega} a(x)(1+\alpha(q(x)+s))^{\beta+1}\left|\partial_{t} u\right|^{r+1} d x d s .
\end{aligned}
$$

and

$$
\begin{aligned}
& \int_{0}^{n T} \int_{\Omega} a(x)(1+\alpha(q(x)+s))^{\beta}\left|\partial_{t} u\right|^{2} d x d s \\
& \leq C \epsilon^{-\frac{2}{r-1}}+\frac{2 \epsilon}{r+1} \int_{0}^{n T} \int_{\Omega} a(x)(1+\alpha(q(x)+s))^{\beta+1}\left|\partial_{t} u\right|^{r+1} d x d s
\end{aligned}
$$

We choose $\epsilon$ such that

$$
\lambda \delta_{0}-k_{3} \epsilon\left(\frac{p-2 r}{p-r-1}+\frac{2}{r+1}\right) \geq \frac{\lambda \delta_{0}}{2}
$$


So there exists a positive constant $C_{0}$ such that

$$
\begin{aligned}
& X(n T)+\frac{1-k \alpha(1+\beta)}{4} \int_{0}^{n T} \int_{\Omega}(1+\alpha(q(x)+s))^{\beta}\left(|\nabla u|^{2}+\left|\partial_{t} u\right|^{2}\right) d x d s \\
& +\delta_{0} \int_{0}^{n T} \int_{\Omega} a(x)(1+\alpha(q(x)+s))^{\beta-2}|u|^{2} d x d s \\
& +\frac{\lambda \delta_{0}}{2} \int_{0}^{n T} \int_{\Omega} a(x)(1+\alpha(q(x)+s))^{\beta+1}\left|\partial_{t} u\right|^{r+1} d x d s \leq C_{0} I_{1}, \text { for all } n \in \mathbb{N} .
\end{aligned}
$$

Therefore we obtain

$$
\begin{aligned}
& \frac{1-k \alpha(1+\beta)}{4} \int_{0}^{+\infty} \int_{\Omega}(1+\alpha(q(x)+s))^{\beta}\left(|\nabla u|^{2}+\left|\partial_{t} u\right|^{2}\right) d x d s \\
& +\delta_{0} \int_{0}^{+\infty} \int_{\Omega} a(x)(1+\alpha(q(x)+s))^{\beta-2}|u|^{2} d x d s \\
& +\frac{\lambda \delta_{0}}{2} \int_{0}^{+\infty} \int_{\Omega} a(x)(1+\alpha(q(x)+s))^{\beta+1}\left|\partial_{t} u\right|^{r+1} d x d s \leq C_{0} I_{1} \text {, for all } n \in \mathbb{N} .
\end{aligned}
$$

Now using the weighted energy estimate (2.3), we infer that

$$
\begin{aligned}
& E_{\varphi}(u)(t)=\frac{1}{2} \int_{\Omega}(1+\alpha(q(x)+s))^{\beta+1}\left(|\nabla u|^{2}+\left|\partial_{t} u\right|^{2}\right) d x \\
& \leq E_{\varphi}(u)(0)+\alpha(\beta+1) \int_{0}^{\infty} \int_{\Omega}(1+\alpha(q(x)+s))^{\beta}\left(|\nabla u(s)|^{2}+\left|\partial_{t} u(s)\right|^{2}\right) d x d s \\
& \leq C_{1} I_{1},
\end{aligned}
$$

for some positive constant $C_{1}$. The sought estimate follows from the estimate above and the fact that

$$
(1+\alpha t)^{\beta+1} E_{u}(t) \leq E_{\varphi}(u)(t) .
$$

This finishes the proof of theorem 2 .

\section{Proof of Theorem 3}

5.1. Preliminary results. Throughout this section we use the following notations: Let $\beta$ be a real number such that $0<1+\beta<\tau$, where

$$
\tau_{1}=\frac{2 r \delta_{0}^{\frac{r^{2}+1}{r}}(\lambda+1)^{r-1}(r+1)^{r}}{\delta_{0}^{\frac{r+1}{r}}+\delta_{0}^{r}(\lambda+1)^{r-1}(r+1)^{r}\left(r \delta_{0}(\lambda+1)(r+1)+2 \delta_{0}^{\frac{1}{r}}\right)},
$$

$\lambda$ any positive constant such that $\lambda<1$ and

$$
\delta_{0}=(\lambda+1)^{\frac{r^{2}}{r^{2}-1}}(r+1)^{-\frac{r}{r-1}} .
$$

Let $R>0$, we take $\varphi(s)=(R+\alpha s)^{\beta+1}$ where

$$
\alpha=\frac{r k^{r}(r+1)+\delta_{0}^{\frac{1}{r}}}{k^{r} \delta_{0}^{\frac{1}{r}}(r+1)\left(r-\tau_{1}\right)},
$$

and

$$
k=(1+\lambda)(r+1) \delta_{0} .
$$


Finally, let $\psi \in C_{c}^{\infty}\left(\mathbb{R}^{d}\right)$ such that $0 \leq \psi \leq 1$ and

$$
\psi(x)= \begin{cases}1 & \text { for }|x| \leq L \\ 0 & \text { for }|x| \geq 2 L\end{cases}
$$

From proposition 2 we deduce the following result.

Proposition 5. We assume that Hyp $A$ holds and $(\omega, T)$ geometrically controls $\Omega$. Let $\delta>0, R, R_{0}>L$ and $-1<\beta \leq 0$. There exists $C_{T, \delta}=C\left(T, \delta, R_{0}, R, \alpha, \beta\right)>0$, such that the following inequality

$$
\begin{aligned}
& \int_{t}^{t+T} \int_{\Omega \cap B_{R_{0}}}(R+\alpha s)^{\beta}\left(|u|^{2}+|\nabla u|^{2}+\left|\partial_{t} u\right|^{2}\right) d x d s \\
& \leq C_{T, \delta} \int_{t}^{t+T} \int_{\Omega} a(x)(R+\alpha s)^{\beta}\left(\left|\partial_{t} u\right|^{2}+\left|\partial_{t} u\right|^{2 r}\right) d x d s \\
& +C_{T, \delta} \int_{t}^{t+T} \int_{\Omega} a(x)(R+\alpha s)^{\beta-2}|u|^{2} d x d s \\
& +\delta \int_{t}^{t+T} \int_{\Omega}(R+\alpha s)^{\beta}\left(|\nabla u|^{2}+\left|\partial_{t} u\right|^{2}\right) d x d s,
\end{aligned}
$$

holds for every $t \geq 0$ and for all $u$ solution of (1.1) with initial data $\left(u_{0}, u_{1}\right)$ in $H_{0}^{1}(\Omega) \cap$ $H^{2}(\Omega) \times H_{0}^{1}(\Omega)$.

As in the proof of theorem we need to define and to show an estimate for an auxiliary function $X(t)$.

Lemma 4. Let $u$ be a solution of (1.1) with initial data in $H_{0}^{1}(\Omega) \cap H^{2}(\Omega) \times H_{0}^{1}(\Omega)$ such that. We set $\chi=1-\psi$ and

$$
\begin{aligned}
& X(t)=\int_{\Omega}(R+\alpha t)^{\beta} \chi^{2}(x) u(t) \partial_{t} u(t) d x+\frac{k_{1}}{2} \int_{\Omega}(R+\alpha t)^{\beta-1} a(x)|u(t)|^{2} d x \\
& +\int_{\Omega} a(x)(R+\alpha t)^{\beta-r+1}|u(t)|^{r+1} d x+\frac{k}{2} \int_{\Omega}(R+\alpha t)^{\beta+1}\left(|\nabla u|^{2}+\left|\partial_{t} u\right|^{2}\right) d x,
\end{aligned}
$$

where $k_{1}$. Then

$$
\begin{aligned}
& X(t+T)-X(t)+\frac{2-k \alpha(1+\beta)}{2} \int_{t}^{t+T} \int_{\Omega}(R+\alpha s)^{\beta}\left(|\nabla u|^{2}+\left|\partial_{t} u\right|^{2}\right) d x d s \\
& +\left(\frac{k_{1} \alpha(1-\beta)}{4}-\frac{\beta^{2} \alpha^{2}}{\epsilon_{0} \epsilon}\right) \int_{t}^{t+T} \int_{\Omega} a(x)(R+\alpha s)^{\beta-2}|u(t)|^{2} d x d s \\
& +\lambda \delta_{0} \int_{t}^{t+T} \int_{\Omega} a(x)(R+\alpha s)^{\beta+1}\left|\partial_{t} u\right|^{r+1} d x d s \\
& \leq\left(3+\left\|\nabla \chi^{2}\right\|_{\infty}\right) \int_{t}^{t+T} \int_{\Omega \cap B_{2 L}}(R+\alpha s)^{\beta}\left(|u|^{2}+|\nabla u|^{2}+\left|\partial_{t} u\right|^{2}\right) d x d s \\
& +\left(\frac{2}{\epsilon_{0}}+\frac{8 k_{1}}{\alpha(1-\beta)}+\frac{8 \beta^{2} \alpha}{\epsilon_{0}^{2} k_{1}(1-\beta)}\right) \int_{t}^{t+T} \int_{\Omega} a(x)(R+\alpha s)^{\beta}\left|\partial_{t} u\right|^{2} d x d s,
\end{aligned}
$$

for all $t \geq 0$ and any $\lambda>0$. 
Proof. For the proof we have to argue as in the proof of Lemma 3 and to use the fact that

$$
\begin{aligned}
& E_{\varphi}(u)(t+T)+\int_{t}^{t+T} \int_{\Omega} a(x)(R+\alpha s)^{\beta+1}\left|\partial_{t} u\right|^{r+1} d x d s \\
& \leq E_{\varphi}(u)(t)+\frac{(\beta+1) \alpha}{2} \int_{t}^{t+T} \int_{\Omega}(R+\alpha s)^{\beta}\left(|\nabla u|^{2}+\left|\partial_{t} u\right|^{2}\right) d x d s .
\end{aligned}
$$

5.2. Proof of Theorem 3. For the proof we have to proceed as in the proof of theorem 2 and to use the finite speed propagation property and the fact that the support of the initial data is contained in $B_{R}$ to show that

$$
\begin{aligned}
& \int_{0}^{\infty}(R+\alpha s)^{\beta} \int_{\Omega} a(x)\left|\partial_{t} u\right|^{2 r} d x d s \leq C \epsilon^{-\frac{p-2 r}{r-1}}\|a\|_{L^{\infty}}\left(\left\|u_{0}\right\|_{H^{2}}^{2}+\left\|u_{1}\right\|_{H^{1}}^{2}+\left\|u_{1}\right\|_{H^{1}}^{2 r}\right) \\
& +\frac{\epsilon(p-2 r)}{p-r-1} \int_{0}^{\infty} \int_{\Omega} a(x)(R+\alpha s)^{\beta+1}\left|\partial_{t} u\right|^{r+1} d x d s,
\end{aligned}
$$

and

$$
\int_{0}^{\infty}(R+\alpha s)^{\beta} \int_{\Omega} a(x)\left|\partial_{t} u\right|^{2} d x d s \leq C \epsilon^{-\frac{2}{r-1}}+\frac{2 \epsilon}{r+1} \int_{0}^{\infty}(R+\alpha s)^{\beta+1} \int_{\Omega} a(x)\left|\partial_{t} u\right|^{r+1} d x d s,
$$

for some positive constant $C$ and for all $\epsilon>0$.

\section{REFERENCES}

[1] J. Bae, M. Nakao, Energy decay for the wave equation with boundary and localized dissipations in exterior domains, Math. Nachr. 278, No. 7-8, $771-783$ (2005).

[2] C. Bardos, G. Lebeau, J. Rauch, Sharp sufficient conditions for the observation, control, and stabilization of waves from the boundary. SIAM J. Control Optimization 30, No.5, 1024-1065 (1992).

[3] N. Burq, P. Gérard, Condition nécessaire et suffisante pour la contrôlabilité exacte des ondes, Comptes Rendus de l'Académie des Sciences - Series I - Mathematics, Volume 325, Issue 7, October 1997, Pages 749-752.

[4] Cavalcanti, Marcelo M.; Dias Silva, Flavio R.; Cavalcanti, Valéria N. Domingos, Uniform decay rates for the wave equation with nonlinear damping locally distributed in unbounded domains with finite measure. SIAM J. Control Optim. 52 (2014), no. 1, 545-580.

[5] W. Dan, Y. Shibata; On a local energy decay of solutions of a dissipative wave equation. Funkcial. Ekvac. 38 (1995), no. 3, 545-568.

[6] M. Daoulatli, Behaviors of the energy of solutions of the wave equation with damping and external force, Journal of Mathematical Analysis and Applications, Volume 389, Issue 1, 1 (2012), 205-225.

[7] M. Daoulatli, Energy decay rates for solutions of the wave equation with linear damping in exterior domain, Evolution Equations and Control Theory (EECT), Vol 5, Issue 1, (2016), 37 - 59.

[8] M. Daoulatli, Behaviors of the energy of solutions of two coupled wave equations with nonlinear damping on a compact manifold with boundary, arXiv:1703.00172.

[9] R. Ikehata, G. Todorova, B. Yordanov; Critical Exponent for Semilinear Wave Equations with SpaceDependent Potential, Funkcialaj Ekvacioj, 52 (2009), 411-435.

[10] R. Ikehata, Energy decay of solutions for the semilinear dissipative wave equations in an exterior domain, Funkcial. Ekvac. 44 (2001) 487-499.

[11] S. Kawashima, M. Nakao, K. Ono, On the decay property of solutions to the Cauchy problem of the semilinear wave equation with a dissipative term, J. Math. Soc. Jpn. 47 (1995) 617-653.

[12] P. D. Lax and R. S. Phillips, Scattering Theory, Pure and Applied Mathematics, 26. Academic Press, Inc., Boston, MA, 1989.

[13] J.L.Lions and W.Strauss, Some non-linear evolution equations, Bull. Soc. Math. France, 93 (1965), 43-96.

[14] K. Mochizuki, Global existence and energy deacy of small solutions to the Kirchhoff equation with linear dissipation localized near infinity, J.Math. Kyoto Univ. 39(1999), p. 347-364. 
[15] Mochizuki, Kiyoshi; Motai, Takahiro, On energy decay-nondecay problems for wave equations with nonlinear dissipative term in $\mathrm{R}^{N}$, J. Math. Soc. Japan 47 (1995), no. 3, 405-421.

[16] M. Nakao, Decay of solutions to the Cauchy problem for the Klein-Gordon equation with a localized nonlinear dissipation, Hokkaido Math. J. 27 (1998), p. 245-271.

[17] M. Nakao, Energy decay to the Cauchy problem for a generalised nonlinear Klein-Gordon equation with a nonlinear dissipative term. Int. J. Dyn. Syst. Differ. Equ. 3 (2011), no. 3, 349-362.

[18] M. Nakao; Jung, Il Hyo, Energy decay for the wave equation in exterior domains with some half-linear dissipation, Differential Integral Equations 16 (2003), no. 8, 927-948.

[19] K. Ono, The time decay to the Cauchy problem for semilinear dissipative wave equations, Adv. Math. Sci. Appl. 9(1999), 243-262.

[20] K. Ono, $\mathrm{L}^{1}$ estimates for dissipative wave equations in exterior domains. J. Math. Anal. Appl. 333 (2007), no. 2, 1079-1092

[21] R. Racke, Non-homogeneous non-linear damped wave equations in unbounded domains, Math. Methods Appl. Sci. 13 (1990), 481-491.

[22] L. Tartar. H-measures, a new approach for studying homogenisation, oscillations and concentration effects in partial differential equations . Proceedings of the Royal Society of Edinburgh. Section A. Mathematics, 115 (3-4):193-230, 1990

[23] G. Todorova, B. Yordanov, The energy decay problem for wave equations with nonlinear dissipative terms in $\mathrm{R}^{n}$. Indiana Univ. Math. J. 56 (2007), no. 1, 389-416.

[24] K. Wakasa, B. Yordanov, On the energy decay for dissipative nonlinear wave equations in one space dimension, JMAA, In press, accepted manuscript, Available online 8 June 2017.

Imam Abdulrahman Bin faisal University, King Saudi Arabia \& University of Carthage, TUNISIA.

E-mail address, M. Daoulatli: moez.daoulatli@infcom.rnu.tn 